\title{
An XFEM/CZM implementation for massively parallel simulations of composites fracture
}

\author{
Guillermo Vigueras $^{\mathrm{a}, \mathrm{b}}$, Federico Sket $^{\mathrm{a}}$, Cristobal Samaniego ${ }^{\mathrm{c}}$, Ling Wu ${ }^{\mathrm{d}}$, Ludovic Noels ${ }^{\mathrm{d}}$, Denny Tjahjanto ${ }^{\mathrm{a}, \mathrm{e}}$, Eva Casoni $^{\mathrm{c}}$, \\ Guillaume Houzeaux $^{\mathrm{c}}$, Ahmed Makradi ${ }^{\mathrm{f}}$, Jon M. Molina-Aldareguia ${ }^{\mathrm{a}}$, Mariano Vazquez ${ }^{\mathrm{c}}$, Antoine Jérusalem ${ }^{\mathrm{a}, \mathrm{g}}$
}

\author{
${ }^{a}$ IMDEA Materials Institute, Getafe, Spain \\ ${ }^{b}$ IMDEA Software Institute, Madrid, Spain \\ ${ }^{c}$ Barcelona Supercomputing Centre, Barcelona, Spain \\ ${ }^{d}$ University of Liège, Liège, Belgium \\ ${ }^{e}$ KTH Royal Institute of Technology, Stockholm, Sweden \\ ${ }^{f}$ CRP Henri Tudor, Luxembourg-Kirchberg, Luxembourg \\ ${ }^{g}$ University of Oxford, Oxford, UK
}

\begin{abstract}
Because of their widely spread use in many industries, composites are the subject of many research campaigns. More particularly, the development of both accurate and flexible numerical models able to capture their intrinsically multiscale modes of failure is still a challenge. The standard finite element method typically requires intensive remeshing to adequately capture the geometry of the cracks and high accuracy is thus often sacrificed in favor of scalability, and vice versa. In an effort to preserve both properties, we present here an extended finite element method (XFEM) for large scale composite fracture simulations. In this formulation, the standard FEM formulation is partially enriched by use of shifted Heaviside functions with special attention paid to the scalability of the scheme. This enrichment technique offers several benefits, since the interpolation property of the standard shape function still holds at the nodes. Those benefits include (i) no extra boundary condition for the enrichment degree of freedom, and (ii) no need for transition/blending regions; both of which contribute to maintain the scalability of the code.

Two different cohesive zone models (CZM) are then adopted to capture the physics of the crack propagation mechanisms. At the intralaminar level, an extrinsic CZM embedded in the XFEM formulation is used. At the interlaminar level, an intrinsic CZM is adopted for predicting the failure. The overall framework is implemented in ALYA, a mechanics code specifically developed for large scale, massively parallel simulations of coupled multi-physics problems. The implementation of both intrinsic and extrinsic CZM models within the code is such that it conserves the extremely efficient scalability of ALYA while providing accurate physical simulations of computationally expensive phenomena. The strong scalability provided by the proposed implementation is demonstrated. The model is ultimately validated against a full experimental campaign of loading tests and X-ray tomography analyses for a chosen very large scale.
\end{abstract}

Keywords:

Composites, XFEM, Cohesive elements, Failure, Large scale parallel simulations

\section{Introduction}

The prediction of structural failure in laminated composites is particularly challenging since both intra and interlaminar failure mechanisms (highly multiscale in nature) contribute to the fracture process [1]. Indeed, a full understanding of fracture mechanisms in composites requires an approach linking microscale deformation features within a ply to its macroscale laminate counterpart. One way to predict mesoscale fracture from numerical simulations is to analyze the microscale deformation mechanisms with constitutive models accounting for the fracture processes ranging from micro-crack initiation to mesocrack propagation. A natural way to achieve this goal is to enrich the finite element model with the so-called cohesive zone method (CZM).

Email address: antoine.jerusalem@eng.ox.ac.uk (Antoine Jérusalem)
The CZM [2, 3] defines a traction between crack lips during the separation process, which is modeled using a traction separation law (TSL). Figures $1 \mathrm{a}$ and $1 \mathrm{~b}$ show two examples of cohesive laws. In both laws, the TSL curve eventually decreases monotonically until reaching zero at a critical opening displacement $\delta_{c}$ to model a progressive damage of the material. The energy dissipated during this process corresponds to the fracture energy $G_{c}$. The TSL for quasi-brittle materials is thus related to the breaking of atomic bonds which is characterized by two physical parameters: the material strength $\sigma_{c}$ and the fracture energy $G_{c}$.

In most of the finite element applications of the CZM, the cohesive elements are inserted at the beginning of the simulation, in which case the TSL is decomposed into an initial loading phase, followed by an unloading phase once the stress reaches the material strength $\sigma_{c}$ (see Figure 1a). Depending on the model, the unloading can either be reversible or irreversible in case of closure of the crack. Such approach is attractive as it 
can easily be implemented, in particular when the crack path is well defined, e.g. for composite delamination [4, 5]. This socalled "intrinsic" cohesive law has thus extensively been used to model fracture in the matrix phase of composites [6] or to predict the interaction between intralaminar and interlaminar failure mechanisms by inserting cohesive elements in-between all bulk elements [7, 8]. However, such scheme exhibits a strong mesh dependency, alters the structural stiffness and adds spurious stiffness elements in a mesh dependent way, since the initial slope in the reversible part of the TSL does not satisfy the consistency condition. Some authors have tried to mitigate this problem by increasing the initial slope of the TSL [4, 9, 10], but this technique leads to an ill-conditioned stiffness matrix for static simulations or to unacceptable small values of the critical time step for explicit dynamic simulations [11].

In order to avoid the drawbacks inherent to the intrinsic approach, an "extrinsic" cohesive law accounting exclusively for the irreversible phase can be used [12, 13], see Figure 1b. Practically a simulation using such scheme proceeds with a classical finite element approach and cohesive elements are introduced at the interface of bulk elements at the onset of fracture. Although the 3D implementation of this framework [14] is not straightforward due to the mesh topology changes during the computation, it predicts crack propagation with an energy and a crack path that both converge with the mesh size, as shown by Arias et al. [15] and by Molinari et al. [16]. However the implementation complexity increases drastically for parallel framework [17] and can suffer from low scalability unless a graph-based internal structure is used [18, 19].

The difficulties presented by the extrinsic CZM and the limitations of the CZM in its intrinsic form, where a scalable applicability is reduced to cases for which the crack path is well defined, have led some authors to propose hybrid models combining the use of CZM with some numerical methods for simulating damage processes. Research efforts have thus focused on combining intrinsic cohesive elements for delamination, with continuum damage models for ply damage [20, 21, 22]. Although this mesomechanical approach has proved to be successful for some structural configurations [20], the use of two different kinematic representations for interlaminar and intralaminar failure presents some fundamental problems. For example, in the modeling of the interaction between transverse matrix cracks and delamination it is necessary to capture the high stresses at the tip of the transverse crack. However, a mesomechanical model cannot possibly capture this interaction because the elements where the transverse crack is predicted soften without being able to accurately capture the stress field at the interface.

Some other approaches have suggested hybrid discontinuous Galerkin (DG)/CZM approaches [23, 24, 25]. The main feature of the DG method for non-linear solid mechanics is its ability to take into account discontinuities of the unknown field between bulk elements [26, 27, 28, 29, 30]. With the hybrid DG/CZM method, interface elements are therefore inserted between bulk elements at the beginning of the simulation and continuity during the pre-fracture stage is ensured by having recourse to the consistent DG interface terms. An extrinsic co- hesive law can thus be integrated on the already existing interface elements once a fracture criterion is met, without requiring mesh topology changes. This approach has been successfully implemented in a 3D parallel framework for micro-meso simulation of intralaminar fracture in composites [31]. The method has also been used in 2D to study the effect of the fibers shapes on the composite resistance [25]. Such an approach has also been developed for thin homogeneous elastic bodies [32, 33] and for thin homogeneous elasto-plastic structures [34]. However, similarly to the simple CZM approach, the discontinuities defined by the DG/CZM approach suffer from mesh dependency. It might thus be adequate for interlaminar failure simulations, but not for intralaminar failure.

In order to alleviate the mesh-dependency inherent to the intrinsic cohesive approach, the eXtended (or Generalized) Finite Element Method (XFEM) [35] has been used in combination with CZM [36, 37] for predicting fracture mechanisms. XFEM offers the possibility to represent the entire crack geometry independently of the mesh, so that refined meshes are not necessary to model crack growth. Also, XFEM exploits the partition-of-unity property of finite elements [38], which allows local enrichment functions to be easily incorporated into a finite element approximation. A standard approximation is thus enriched in a region of interest by the local functions in conjunction with additional degrees of freedom. For the purpose of fracture analysis, the enrichment functions are the neartip asymptotic fields and a discontinuous function to represent the jump in displacement across the crack line. These features make XFEM an attractive method for fracture prediction since it can flexibly represent the crack path without prior knowledge of the orientation of the crack plane.

Due to its multiscale nature, the simulation of delamination and ply failure results in high computational requirements which solid mechanics codes traditionally do not manage efficiently. Most of the commercial codes cannot efficiently scale in parallel computers when more than hundreds of cores are used (this is especially true when using implicit solvers). Academic codes, on the other hand, have often relied on the need to develop one unique technique of interest for their development, potentially followed by a secondary development phase aimed at scaling it up because of the prohibitive cost of the technique. Led by the Barcelona Supercomputing Center (BSCCNS), Alya [39, 40], on the contrary, has been conceived from the beginning as a massively large scale computational mechanics code aimed at solving Partial Differential Equations (PDEs) in non-structured meshes. Alya exploits the similarities of the PDE-governed problems to solve with high parallel performance (thousands of cores) compressible and incompressible flows, solid mechanics, thermal flows, excitable media or quantum mechanics for transient molecular dynamics [41, 42, 43, 44, 40]. Parallelization is hidden behind a common solver that assembles matrices and residuals and carries out the solution scheme. The scalability of the code thus exclusively depends on one unique set of parallel communication subroutines independently of the physics of the problem.

We thus propose herein an Alya-implementation of XFEM for the simulation of fracture in laminated composite materials. 


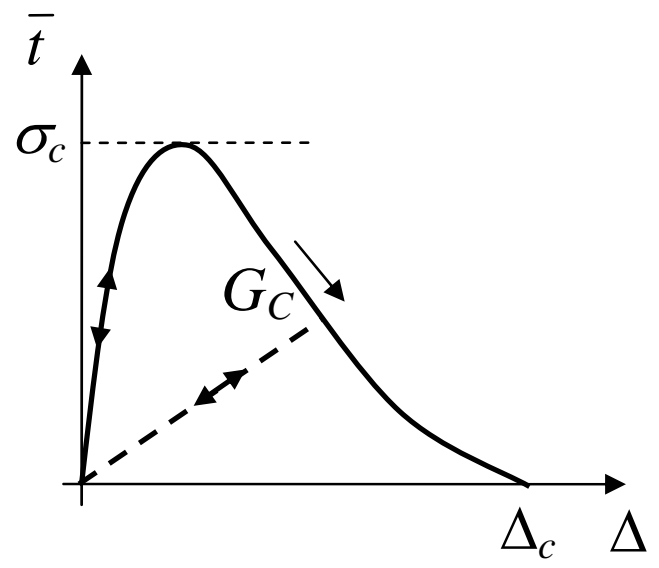

(a) Intrinsic

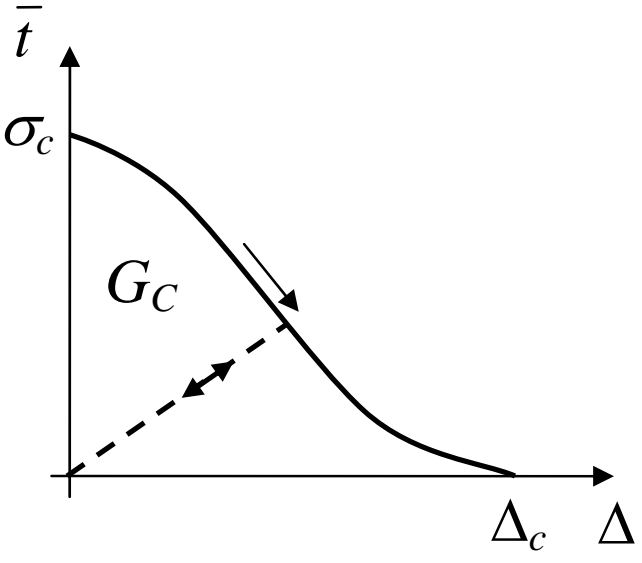

(b) Extrinsic

Figure 1: Cohesive laws

In this approach, the intrinsic CZM is used to model interlaminar fracture, whereas XFEM is combined with the extrinsic CZM to model intralaminar fracture. The models are implemented so as to conserve the extreme scalability of Alya while ensuring the accurate physical description of the material deformation and failure. The details of this scalable implementation are thoroughly described.

The implementation is ultimately validated against experimental open hole tensile tests of laminated carbon fiber reinforced polymers (CFRP) where damage has been thoroughly characterized by X-ray computed tomography (XCT). XCT provides actual 3D information of the damage and microstructure in every ply from a number of X-ray radiographies obtained at different angles in a non-destructive manner. In addition, contrast enhancement was obtained by infiltrating a liquid with a high X-ray absorption coefficient (e.g. ZnI), leading to a complete characterization of damage by matrix cracking and interply delamination [45, 46]. While the damage mechanisms in fiber reinforced polymers are qualitatively well documented in the literature [47, 48, 49, 50, 51, 52], quantitative data on the evolution of matrix cracking and interply delamination with strain is less common [53]. Traditionally, quantitative characterization has been limited by time consuming and destructive serial sectioning microscopy methods or to simpler laminate sequences due to the inability of X-ray radiography to separate the contribution from different plies [54, 53]. These limitations are overcome by XCT, which has been used in this work to follow the development of damage of each individual ply of $[90 /+45 /-45 / 90 / 0]_{s}$ carbon fiber laminates during the tensile deformation. The evaluation was focused on the evolution of crack density and delamination as a function of strain, for direct comparison with the simulation results, which show an excellent agreement.

Section 2 presents the numerical scheme implemented in Alya and Section 3 focuses on implementation specificities required to conserve both the robustness and scalability of Alya. The experimental campaign, i.e. materials mechanical and XCT characterization, is provided in Section 4. The results are discussed in Section 5, and the conclusions of this work are finally given in Section 6 .

\section{Numerical Scheme}

This section describes the standard Galerkin formulation with XFEM enrichment of the solution, and the cohesive laws used for the inter- and intralaminar fracture models. Implementation details of the discretization and integration are also described. See Ref. [23, 24, 31] for more details.

\subsection{Enrichment of solution approximation}

Let $\boldsymbol{X} \in B_{0}$ be a material point in the reference configuration, and $\boldsymbol{x}=\varphi(\boldsymbol{X}) \in B$ be the corresponding point in the deformed configuration, where $\varphi$ is the deformation function. The deformation gradient tensor $\boldsymbol{F}$ is defined as

$$
\boldsymbol{F}=\operatorname{Grad} \boldsymbol{x} .
$$

In a Cartesian basis the components of $\boldsymbol{F}$ are given by

$$
F_{i J}=\frac{\partial x_{i}}{\partial X_{J}} .
$$

Let the undeformed body $B_{0}$ be approximated by a domain $\Omega_{0} \approx B_{0}$, where $\Omega_{0}$ is subdivided into a set of triangular elements $\Omega_{0}^{e}$, such that $\Omega_{0}=\cup_{e} \Omega_{0}^{e}$.

Recall that in a standard finite element method, the solution approximation of nodal displacement $\boldsymbol{u}=\boldsymbol{x}-\boldsymbol{X}$ can be written as

$$
\boldsymbol{u}(\boldsymbol{X}) \approx \boldsymbol{u}^{h}(\boldsymbol{X})=\sum_{a \in I} N^{a}(\boldsymbol{X}) \boldsymbol{u}^{a},
$$

where $I$ is the set of nodes $a$ supporting the domain $\Omega_{0}$, and $N^{a}$ is the shape function corresponding to the nodal support $a$. In a finite element discretization, $N^{a}$ is chosen such that it has a compact support on node $a$, i.e. $N^{a}\left(\boldsymbol{X}^{a}\right)=1$ and $N^{a}\left(\boldsymbol{X}^{b}\right)=0$ for all $b \neq a$. Therefore, the above solution approximation satisfies the Kronecker-delta or interpolation property, $\boldsymbol{u}^{a}=\boldsymbol{u}^{h}\left(\boldsymbol{X}^{a}\right)$. 
In order to account for discontinuities, the solution approximation (3) is enriched using additional interpolation functions capturing the discontinuities (or sharp gradients). Let $\psi(\boldsymbol{X})$ be an enrichment function, such that the enriched solution approximation can be written as

$$
\boldsymbol{u}^{h}(\boldsymbol{X})=\sum_{a \in I} N^{a}(\boldsymbol{X}) \boldsymbol{u}^{a}+\sum_{a \in I} f^{a}(\boldsymbol{X}) \psi(\boldsymbol{X}) \boldsymbol{\alpha}^{a},
$$

where $\boldsymbol{\alpha}^{a}$ is the vector of additional unknowns or degrees of freedom associated with the enriched solution for node $a$, and where $f^{a}(\boldsymbol{X})$ is the corresponding interpolation function that satisfies the partition-of-unity property, i.e.

$$
\sum_{a \in I} f^{a}(\boldsymbol{X})=1
$$

For simplicity, the partition-of-unity function $f^{a}$ is chosen to be the same as the standard finite element shape function, i.e. $f^{a}=N^{a}$. The expression for the enriched approximation of the displacement field thus reads as

$$
\boldsymbol{u}^{h}(\boldsymbol{X})=\sum_{a \in I} N^{a}(\boldsymbol{X})\left(\boldsymbol{u}^{a}+\psi(\boldsymbol{X}) \boldsymbol{\alpha}^{a}\right)
$$

Cracks are growing open interfaces and can thus be represented by two level set functions $\Phi$ and $\Lambda$, describing respectively the path of the crack and the position of the crack tip(s)/edge(s), such that $\Gamma_{0 c}=\{\boldsymbol{X} \mid \Phi(\boldsymbol{X})=0$ and $\Lambda(\boldsymbol{X}) \leq 0\}$. In this case, the level set function $\Phi$ can be described using a signed-distance function as follows:

$$
\Phi(\boldsymbol{X})=\left\|\boldsymbol{X}-\boldsymbol{X}_{\Gamma}\right\| \operatorname{sign}\left(\boldsymbol{n}_{\Gamma} \cdot\left(\boldsymbol{X}-\boldsymbol{X}_{\Gamma}\right)\right),
$$

for all $\boldsymbol{X} \in \Omega_{0}$, where $\boldsymbol{X}_{\Gamma}$ is the projection of $\boldsymbol{X}$ on the crack path $\Gamma_{0 c}$ and $\boldsymbol{n}_{\Gamma}$ is a vector normal (with a continuous orientation along the crack path) to the path at point $\boldsymbol{X}_{\Gamma}$. Meanwhile, the level set function $\Lambda(X)$ is constructed such that its path is orthogonal/perpendicular to the path of $\Phi(\boldsymbol{X})$ at the crack tip(s).

Note that special treatments need to be introduced to deal with singularity at the crack tip. Such approaches generally include additional enrichment functions at the crack tip based on the elastic fracture mechanics model in order to deal with the singularity associated with the crack tip. Alternatively, the cohesive law model typically used in quasi-brittle and ductile materials can be used to solve both the crack opening and the crack tip singularity problem without additional enrichment [55]. Moreover in the cohesive crack approach, cracks are virtually extended up to the edge of the partitioned elements, such that locally (element-wise), the crack path can be considered as a closed interface. More details on the cohesive crack model will be presented in Section 2.3

Assuming closed interfaces at the element level, the Heaviside enrichment function $H$ for fully partitioned elements (with $\Lambda \leq 0$ ) is given by

$$
\psi(\boldsymbol{X})=H(\Phi(\boldsymbol{X}))= \begin{cases}1 & \forall \Phi(\boldsymbol{X})>0 \\ 0 & \forall \Phi(\boldsymbol{X})<0\end{cases}
$$

as illustrated in Figure 2 a. The effective enrichment function of node $a$ is then obtained by $N^{a}(\boldsymbol{X}) H(\boldsymbol{X})$, see Figure $2 \mathrm{p}$. However, the above enrichment function does not satisfy the Kroneckerdelta (or interpolation) property at nodes:

$$
\boldsymbol{u}^{h}\left(\boldsymbol{X}^{a}\right)=\boldsymbol{u}^{a}+H\left(\boldsymbol{X}^{a}\right) \boldsymbol{\alpha}^{a} \neq \boldsymbol{u}^{a} .
$$

To overcome this issue, a shifted Heaviside function $\tilde{H}^{a}(\boldsymbol{X})=$ $H(\boldsymbol{X})-H\left(\boldsymbol{X}^{a}\right)$ is applied in place of the original Heaviside enrichment function, such that the enriched solution approximation of Equation (6) reads [55]:

$$
\boldsymbol{u}^{h}(\boldsymbol{X})=\sum_{a \in I} N^{a}(\boldsymbol{X})\left(\boldsymbol{u}^{a}+\tilde{H}^{a}(\boldsymbol{X}) \boldsymbol{\alpha}^{a}\right) .
$$

The enriched solution approximation in Equation (10) guarantees the interpolation property of diplacement $\boldsymbol{u}^{h}\left(\boldsymbol{X}^{a}\right)=\boldsymbol{u}^{a}$, whereas the effective jump in the displacement field across the crack path $\Gamma_{0 c}$ is given by $\llbracket \boldsymbol{u}^{h}(\boldsymbol{X}) \rrbracket=\sum_{a \in I} N^{a}(\boldsymbol{X}) \boldsymbol{\alpha}^{a}$, for all $\boldsymbol{X} \in \Gamma_{0 c}$, as indicated in Figure $2 \mathrm{~d}$.

Because the effect of discontinuity is local within a limited region around the crack, Heaviside enrichments can be applied partially, i.e. only on a particular domain $\Omega_{0 c} \subset \Omega_{0}$ in the neighborhood of the crack path $\Gamma_{0 c}$. Another advantage of this formulation is that there is no need for special treatments in the blending/transition elements situated in-between enriched and non-enriched elements: the elements in the transition region can be treated as standard elements. Indeed, smooth transition between the displacement unknowns of the enriched element and of the standard elements is guaranteed, thanks to the interpolation property of the shifted shape function, $\boldsymbol{u}^{a}=\boldsymbol{u}^{h}\left(\boldsymbol{X}^{a}\right)$. Note that blending elements could potentially still be needed in other situations, such as general stress intensity factor calculations.

\subsection{Governing equations}

The equation of balance of momentum with respect to the reference configuration can be written as

$$
\operatorname{Div} \boldsymbol{P}+\boldsymbol{b}_{0}=\rho_{0} \ddot{\boldsymbol{u}}, \forall \boldsymbol{X} \in B_{0},
$$

where $\rho_{0}$ is the mass density (with respect to the reference volume) and Div is the divergence operator with respect to the reference configuration. Tensor $\boldsymbol{P}$ and vector $\boldsymbol{b}_{0}$ stand for, respectively, the first Piola-Kirchhoff stress and the distributed body force on the undeformed body. Prescribed displacements and tractions are applied on the reference boundary $\Gamma_{0}=\Gamma_{d 0} \cup \Gamma_{n 0}$, where $\Gamma_{d 0}$ and $\Gamma_{n 0}$ correspond to the Dirichlet and Neumann boundary conditions, respectively, as follows:

$$
\begin{gathered}
\boldsymbol{u}=\overline{\boldsymbol{u}}, \quad \forall \boldsymbol{X} \in \Gamma_{d 0}, \\
\boldsymbol{P} \cdot \boldsymbol{n}_{0}=\overline{\boldsymbol{t}}_{0}, \quad \forall \boldsymbol{X} \in \Gamma_{n 0} .
\end{gathered}
$$

where $\boldsymbol{n}_{0}$ is the normal to the reference surface at the corresponding boundary.

The weak form of balance of the momentum Equation (11) can be formulated as

$$
\int_{B_{0}} \operatorname{Div} \boldsymbol{P} \cdot \boldsymbol{w} \mathrm{d} V_{0}+\int_{B_{0}} \boldsymbol{b}_{0} \cdot \boldsymbol{w} \mathrm{d} V_{0}=\int_{B_{0}} \rho_{0} \ddot{\boldsymbol{u}} \cdot \boldsymbol{w} \mathrm{d} V_{0} .
$$




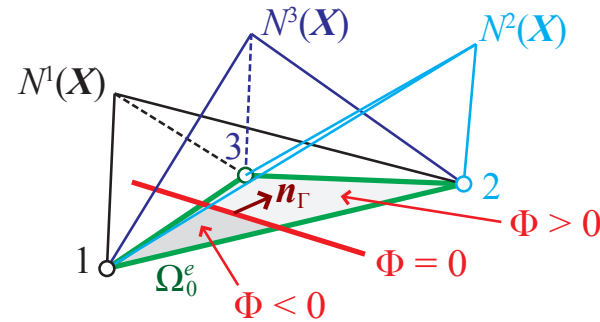

(a) Standard FE shape functions

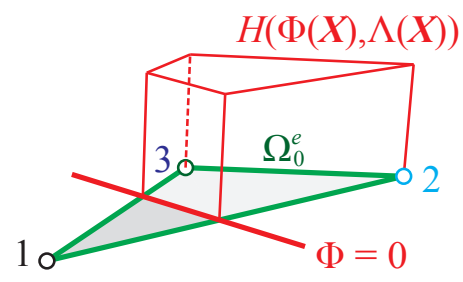

(b) Heaviside function for crack

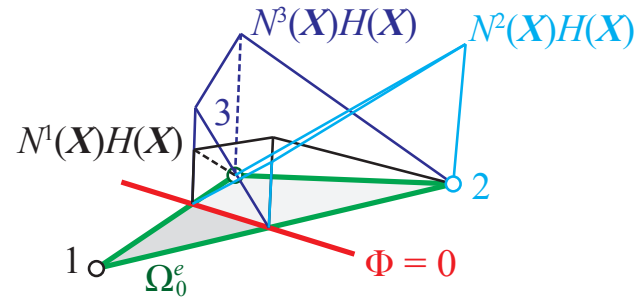

(c) FE enrichment functions by heaviside

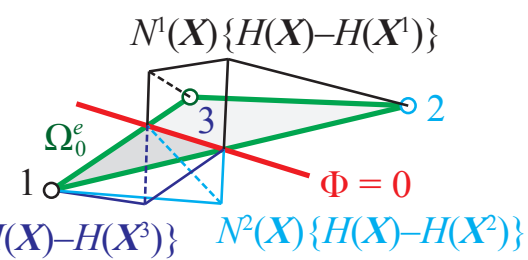

(d) FE enrichment functions with shifting

Figure 2: Finite element shape functions $N^{a}(\boldsymbol{X})$ and the enriched functions $N^{a}(\boldsymbol{X}) \psi(\boldsymbol{X})$ using Heaviside function as enrichment

for any arbitrary admissible virtual displacement $\boldsymbol{w}$.

By making use of the finite element approximation of the enriched solution (see Equation $(10)$ ) for both the discretized solution and the discretized virtual displacement, the XFEM formulation for the balance of momentum Equation (14) thus reads as follows: find the vector of unknowns $z$ made of all $\boldsymbol{u}^{a} \in \mathbb{R}^{3}$ and $\boldsymbol{\alpha}^{a} \in \mathbb{R}^{3}$ of all nodes $a$ such that:

$$
\left[\begin{array}{ll}
\boldsymbol{M}_{u u} & \boldsymbol{M}_{\boldsymbol{u} \alpha} \\
\boldsymbol{M}_{\boldsymbol{\alpha} \boldsymbol{u}} & \boldsymbol{M}_{\boldsymbol{\alpha} \alpha}
\end{array}\right] \cdot \ddot{z}+\left(\begin{array}{c}
\boldsymbol{f}_{\mathrm{int}, \boldsymbol{u}} \\
\boldsymbol{f}_{\mathrm{int}, \boldsymbol{\alpha}}
\end{array}\right)=\left(\begin{array}{c}
\boldsymbol{f}_{\mathrm{ext}, \boldsymbol{u}} \\
f_{\mathrm{ext}, \boldsymbol{\alpha}}
\end{array}\right),
$$

where $\boldsymbol{M}_{\boldsymbol{u} u}, \boldsymbol{M}_{\boldsymbol{u} \boldsymbol{\alpha}}, \boldsymbol{M}_{\boldsymbol{\alpha} \boldsymbol{u}}, \boldsymbol{M}_{\boldsymbol{\alpha} \alpha}, \boldsymbol{f}_{\text {int } \boldsymbol{u}}, \boldsymbol{f}_{\text {int }, \boldsymbol{\alpha}}, \boldsymbol{f}_{\text {ext }, \boldsymbol{u}}$ and $\boldsymbol{f}_{\text {ext }, \boldsymbol{\alpha}}$ are, respectively, the mass matrices, vectors of internal and external forces for the unknown displacements and additional degrees of freedom, along with the cross-terms for the mass matrices. These quantities are constructed and assembled from the corresponding element quantities $\boldsymbol{M}_{\boldsymbol{u} \boldsymbol{u}}^{e}, \boldsymbol{M}_{\boldsymbol{u} \boldsymbol{\alpha}}^{e}, \boldsymbol{M}_{\boldsymbol{\alpha} \boldsymbol{u}}^{e}, \boldsymbol{M}_{\alpha \boldsymbol{\alpha}}^{e}, \boldsymbol{f}_{\text {int, } \boldsymbol{u}}^{e}, \boldsymbol{f}_{\text {int, } \boldsymbol{\alpha}}^{e}$, $\boldsymbol{f}_{e x t, \boldsymbol{u}}^{e}$ and $\boldsymbol{f}_{\text {ext }, \boldsymbol{\alpha}}^{e}$, which are the corresponding mass matrix, internal force vector and external force vectors.

In the above expression, the components $M_{u u}^{e, i a k b}, M_{u \alpha}^{e, i a k b}$, $M_{\alpha u}^{e, i a k b}$, and $M_{\alpha \boldsymbol{\alpha}}^{e, i a k b}$ of the elementary mass matrix are given by

$$
\begin{aligned}
M_{\text {uu }}^{\text {e,iakb }} & =\int_{\Omega_{0}^{e}} \rho_{0} \delta_{i k} N^{a} N^{b} \mathrm{~d} V_{0}, \\
M_{\boldsymbol{u \alpha}}^{\text {e,iakb }} & =\int_{\Omega_{0}^{e}} \rho_{0} \delta_{i k} N^{a}\left(N^{b} \tilde{H}^{b}\right) \mathrm{d} V_{0}, \\
M_{\boldsymbol{\alpha u}}^{e, i a k b} & =\int_{\Omega_{0}^{e}} \rho_{0} \delta_{i k}\left(N^{a} \tilde{H}^{a}\right) N^{b} \mathrm{~d} V_{0}, \\
M_{\boldsymbol{\alpha} \boldsymbol{\alpha}}^{e, i a k b} & =\int_{\Omega_{0}^{e}} \rho_{0} \delta_{i k}\left(N^{a} \tilde{H}^{a}\right)\left(N^{b} \tilde{H}^{b}\right) \mathrm{d} V_{0} .
\end{aligned}
$$

where $\delta_{i k}$ is the Kronecker symbol. The components $f_{\text {int, } \boldsymbol{u}}^{e, i a}$ and $f_{\text {int, } \boldsymbol{\alpha}}^{e, i a}$ of the elementary internal force vector are given by

$$
\begin{aligned}
f_{\text {int }, \boldsymbol{u}}^{e, i a} & =\int_{\Omega_{0}^{e}} P_{i J} N_{, J}^{a} \mathrm{~d} V_{0}, \\
f_{\text {int }, \boldsymbol{\alpha}}^{e, i a} & =\int_{\Omega_{0}^{e}} P_{i J} \tilde{H}^{a} N_{, J}^{a} \mathrm{~d} V_{0}+\int_{\Gamma_{0 c}^{e}} \tilde{t}_{i}^{c} N^{a} \mathrm{~d} S_{0},
\end{aligned}
$$

where $\tilde{\boldsymbol{t}}^{c}$ is the traction at the crack surface ${ }^{1}$. The components of the external force vector $f_{\mathrm{ext}, \boldsymbol{u}}^{e, i a}$ and $f_{\mathrm{ext}, \boldsymbol{\alpha}}^{e, i a}$ are given by

$$
\begin{aligned}
& f_{\text {ext, } \boldsymbol{u}}^{e, i a}=\int_{\Gamma_{0 t}^{e}} \bar{t}_{0 i} N^{a} \mathrm{~d} S_{0}+\int_{\Omega_{0}^{e}} b_{0 i} N^{a} \mathrm{~d} V_{0}, \\
& f_{\text {ext }, \boldsymbol{\alpha}}^{e, i a}=\int_{\Gamma_{0 t}^{e}} \bar{t}_{0 i}\left(N^{a} \tilde{H}^{a}\right) \mathrm{d} S_{0}+\int_{\Omega_{0}^{e}} b_{0 i}\left(N^{a} \tilde{H}^{a}\right) \mathrm{d} V_{0} .
\end{aligned}
$$

The spatial integration in the equations above are done by Gauss quadrature. In the case of a cracked element, the element is cut by a planar interface. This partition results in a failure of the standard Gauss quadrature integration method, as there is no guarantee to have at least one Gauss point on each side of the crack. In order to solve this issue, the element is decomposed into subelements. For example, a cracked tetrahedron is split into a tetrahedral and a pentahedral subelement, in turn subdivided into tetrahedral elements, whereas a cracked hexahedron can be split into a combination of prisms and hexahedra. The numerical integrations on the partitioned element can then be performed using standard Gauss quadrature over the (smooth and continuous) subelements.

In addition, to evaluate the integrals over surfaces/paths Gauss integration points are also defined on surfaces/boundaries. This

\footnotetext{
${ }^{1}$ Note that Nanson's formula should actually be used to relate the traction in the current configuration to the one in the reference configuration. However, matrix cracking almost exclusively occurs at small strain, thus justifying the approximation.
} 
is specifically the case when evaluating cohesive traction on the crack surface, or when applying Neumann boundary conditions. Due to the formation of interfaces (or cracks), additional Gauss integration points are also defined on the (partitioning) interface $\Gamma_{0 c}$ (which is a triangle for tetrahedral elements, or either a triangular or a quadrilateral element for hexahedral elements).

Finally, the time integration of Equation $(15)$ is done by use of the explicit form of the generalized Newmark algorithm, see Ref. [40] for more details.

\subsection{Constitutive model}

As commonly done, simple orthotropic linear elasticity (with adequate rotation with respect to the fiber orientation) is used for the bulk constitutive model, with fiber orientation specific to each ply. This section thus focuses on the interlaminar and intralaminar failure constitutive models. The formulations of the proposed models are briefly summarized in the following, see Camacho and Ortiz [12] for more details.

Both models define the traction-separation law, $\tilde{\boldsymbol{t}}^{c}\left(\boldsymbol{\delta}_{\Gamma}\right)$, which is required in order to describe the evolution of cohesive crack. The jump/discontinuity in the displacement field (crack opening) $\delta_{\Gamma}$ is obtained from

$$
\boldsymbol{\delta}_{\Gamma}(\boldsymbol{X})=\llbracket \boldsymbol{u}^{h}(\boldsymbol{X}) \rrbracket=\sum_{a \in I_{c}} N^{a}(\boldsymbol{X}) \boldsymbol{\alpha}^{a},
$$

for all $\boldsymbol{X} \in \Gamma_{0 c}$, where the crack surface is $\Gamma_{0 c}$ defined by $I_{c}$, a subset of $I$.

A scalar representing the effective opening of displacement $\delta_{\Gamma}$ can be defined as

$$
\tilde{\delta}_{\Gamma}=\sqrt{\lambda^{2}\left(\delta_{\Gamma, s}\right)^{2}+\left(\delta_{\Gamma, n}\right)^{2}} .
$$

where $\lambda$ determines the effective contribution/weight of the norm of the sliding/tangential component $\delta_{\Gamma, s}$ of the displacement opening with respect to the norm of its normal component $\delta_{\Gamma, n}$. The interface traction $\tilde{\boldsymbol{t}}^{c}$ corresponding to the above effective displacement opening can then be expressed as

$$
\tilde{\boldsymbol{t}}^{c}=\frac{\tilde{t}^{c}}{\tilde{\delta}_{\Gamma}}\left(\lambda^{2} \delta_{\Gamma, s} \boldsymbol{s}_{\Gamma}+\delta_{\Gamma, n} \boldsymbol{n}_{\Gamma}\right)
$$

where $\tilde{t}^{c}$ is a scalar representing the effective interface traction, and where $\boldsymbol{s}_{\Gamma}$ and $\boldsymbol{n}_{\Gamma}$ are the local tangential and normal basis vectors on the crack surface.

\subsubsection{Interlaminar failure}

The Rose-Ferrante intrinsic cohesive law is used to model the interlaminar delamination process [13]. The corresponding elements are introduced from the beginning as a preprocessing steps. This choice is rationalized by the fact that the delamination is expected to occur between the plies. This intrinsic law describes the envelope of the relation between the effective traction $\tilde{t}^{c}$ and the corresponding effective displacement separation $\tilde{\delta}_{\Gamma}$ upon loading (or crack opening):

$$
\tilde{t}^{c}=e t_{\text {crit }} \frac{\tilde{\delta}_{\Gamma}}{\delta_{\text {crit }}} \exp \left(-\frac{\tilde{\delta}_{\Gamma}}{\delta_{\text {crit }}}\right), \quad \text { if } \tilde{\delta}_{\Gamma}=\tilde{\delta}_{\max } \text { and } \dot{\tilde{\delta}}_{\Gamma} \geq 0
$$

where $e=\exp (1), t_{\text {crit }}$ and $\delta_{\text {crit }}$ are, respectively, the critical effective cohesive traction and the critical/characteristic displacement opening, and $\tilde{\delta}_{\max }$ is the maximum attained effective displacement opening. Note that this formulation assumes that Mode I and Mode II have the same properties as a first approximation; other models can be straightforwardly added if necessary. Additionally, even if this approximation is acknowledgedly rough (a ratio of 1:5 is generally observed between the cohesive energy of both modes), the results in the following sections exhibit a very good fit with experiments, thus justifying further this initial assumption.

Upon unloading (or upon subsequent loading), the tractionseparation relationship follows a linear elastic curve to the origin,

$$
\tilde{t}^{c}=\frac{\tilde{t}_{\max }}{\tilde{\delta}_{\max }} \tilde{\delta}_{\Gamma}, \quad \text { if } \tilde{\delta}_{\Gamma}<\tilde{\delta}_{\max } \text { or } \dot{\tilde{\delta}}_{\Gamma}<0,
$$

where $\tilde{t}_{\max }=\tilde{t}^{c}\left(\tilde{\delta}_{\max }\right)$. For practical purpose, the traction is set to be zero after a certain amount of opening $\delta_{\infty}$, i.e. $\tilde{t}^{c}\left(\tilde{\delta}_{\Gamma}\right)=0$ if $\tilde{\delta}_{\Gamma} \geq \delta_{\infty}$.

The fracture energy (or fracture toughness), defined as $G_{c}=$ $\int_{0}^{\infty} \tilde{t}^{c} \mathrm{~d} \tilde{\delta}_{\Gamma}$, for the Smith-Ferrante cohesive model is finally given by

$$
G_{c}=e t_{\text {crit }} \delta_{\text {crit }},
$$

\subsubsection{Intralaminar failure}

Intralaminar failure should theoretically account for matrix failure, matrix-fiber failure and fiber failure altogether [31]. The latter will generally occur in situations where the laminates are loaded in the direction of the fibers. This can easily be implemented by projecting the stress tensor in the fiber direction, defining a stress threshold at which the fibers would fail and open a crack perpendicular to this direction. However, one of the current challenges of intralaminar failure is related to the remaining failure modes (matrix and matrix-fiber), where continuum models fail at capturing the correct crack propagation orientation [56]. This is due to the fact that such a crack is actually physically constrained by the fiber direction, phenomenon impossible to capture by means of a regular continuum approach. To this end, we focus here our work on these two failure modes and make sure in the following that none of the failure modes involved in the proposed configurations immediately leads to fiber failure. Extension to fiber failure could eventually be considered by simply adding another nodal degree of freedom for the corresponding crack description.

In this case, the cohesive elements are introduced on-thefly in the then-enriched elements following a modified Rankine principal tensile stress criterion projected on the plane perpendicular to the fibers, i.e. when the maximum of the eigenvalues of the Cauchy stress within such plane reaches a threshold $\sigma_{\max }$ (see also Section 3.2). The crack normal $\boldsymbol{n}_{\Gamma}$ is then chosen as the corresponding eigenvector, and crack continuity with neighboring elements is enforced when applicable, see Section 3. A linearly-decreasing cohesive law is used to simulate the intralaminar damage since this model is more suitable for an 
extrinsic cohesive zone model, where the cohesive law is applied only when the crack/discontinuity is introduced.

In the linearly-decreasing cohesive model, the effective traction $\tilde{t}^{c}$ upon loading (crack opening) is given by

$$
\tilde{t}^{c}=t_{\text {crit }}\left(1-\frac{\tilde{\delta}_{\Gamma}}{\delta_{\infty}}\right), \quad \text { if } \tilde{\delta}_{\Gamma}=\tilde{\delta}_{\max } \text { and } \dot{\tilde{\delta}}_{\Gamma} \geq 0,
$$

with $\delta_{\infty}$ the maximum level of displacement opening where the traction is non-zero $\left(\tilde{t}^{c}=0\right.$ for $\left.\tilde{\delta}_{\Gamma}>\delta_{\infty}\right)$. Note that the above traction-separation relation is valid only for positive opening, $\tilde{\delta}_{\Gamma}>0$. Upon unloading/reloading, the traction-separation relation follows the elastic unloading path described by Equation (23). The fracture toughness associated with the linearlydecreasing cohesive law is given by

$$
G_{c}=\frac{1}{2} t_{\text {crit }} \delta_{\infty}
$$

\section{Robustness and scalability requirements}

The framework described above was implemented in Alya by adding the corresponding degrees of freedom to the solid mechanics module of the code. This solid mechanics module was implemented specifically so as to retain the extremely high scalability of Alya while being flexible enough for future extension such as the one proposed here [40]. However, the numerical scheme proposed here does not easily lend itself to the scalability requirements of Alya, or to a strong robustness. To this end, some special attention was paid to important details of the implementations. They are summarized in the following.

\subsection{Local enriched element remeshing}

As described in Section 2.2, enriched elements are split in subelements on each side of the crack path. This step is done exclusively locally by adding the additional nodes only at the element level while the neighboring elements remain unaware of the splitting and of these additional nodes. The evaluation of the (original) element mass and stiffness matrices, and corresponding right-hand side vector is then done by summing up the contributions of all the subelement Gauss points instead of the original ones, and their sizes remain conditioned by the original element nodes.

It is important to note that the above element decomposition is merely done for the purpose of Gauss integration method and does not create new degrees of freedom (or unknowns). In other words, there is no need to inform the neighboring elements, or processors in the case of parallel simulations. As the number of enriched elements is in general largely outnumbered by the number of regular elements, the balancing of the number of Gauss point evaluations per processor and thus the overall code scalability remain unaffected.

\subsection{Non-locality of the crack propagation}

Based on the modified Rankine principal tensile stress criterion used here, the normal in the reference configuration of the crack/discontinuity plane within an element, $\boldsymbol{n}_{\Gamma}$, is set by the direction of the maximum principal tensile stress in the plane perpendicular to the fiber direction, i.e the eigenvector associated with the most positive eigenvalue of the projected stress tensor,

$$
\boldsymbol{n}_{\Gamma}=\frac{\boldsymbol{F}^{T} \cdot \boldsymbol{v}_{\max }}{\left\|\boldsymbol{F}^{T} \cdot \boldsymbol{v}_{\max }\right\|},
$$

where $\boldsymbol{v}_{\max }$ is the eigenvector associated with the most positive eigenvalue in the plane perpendicular to the fiber direction. Note that this finite deformation formulation can actually be easily simplified to small deformation $\left(\boldsymbol{n}_{\Gamma}=\boldsymbol{v}_{\max }\right)$ in the case of composites laminates as failure occurs generally at strains of only a few percents. In order to improve the accuracy, the Cauchy stress tensor $\sigma$ used for the eigenproblem is averaged over the element in orded to improve the accuracy of the prediction. This is done in Alya by extrapolating the stress values at the nodes, assembling them with the other elements (and processors when required) as it is done in a postprocessing step for field visualization purposes. Doing so, each element stress considered is the average of these node values and the stress averaging thus includes the neighboring elements. Without this non-local step, crack orientation is only conditioned by the local element field, which was observed to lead to spurious curvature of the crack.

Once the crack orientation is defined, it needs to be attached, when applicable, to the cracks of the neighboring elements. Let $\boldsymbol{p}^{i}$ be the mid-point of the intersection line-segment between the element facet $f_{i}$ and the existing discontinuity plane from the adjacent element, as illustrated in Figure 3 . When the modified Rankine criterion is fulfilled, the crack surface/plane is formed within the element through a point $\boldsymbol{p}_{\Gamma}$, which is defined as

$$
\boldsymbol{p}_{\Gamma}=\frac{1}{N_{i}} \sum_{i=1}^{N_{i}} \boldsymbol{p}^{i}
$$

with $N_{i}$ the total number of facets of the element that intersect with the existing crack. This above condition is applied to ensure the continuity of the crack path. Although in 3-dimensional cases this condition cannot guarantee the connectivity of the piecewise elementary crack planes across the element faces (see Figure 3, the present scheme should be able to resemble the approximation of the global crack surface [57]).

In case of crack initiation, where there is no existing crack path adjacent elements, the crack position is assumed to form through the center of the element:

$$
\boldsymbol{p}_{\Gamma}=\frac{1}{N_{a}} \sum_{a=1}^{N_{a}} \boldsymbol{X}^{a},
$$

where $\boldsymbol{X}^{a}$ is the coordinate of the nodes, with $N_{a}$ the number of nodes of the element.

\subsection{Topological constraints for crack propagation}

The present discrete/element-wise crack propagation model often encounters severe numerical instability issues. In the cases presented in this work, two crack propagation scenarii have been identified, which lead to unstable crack propagation. 


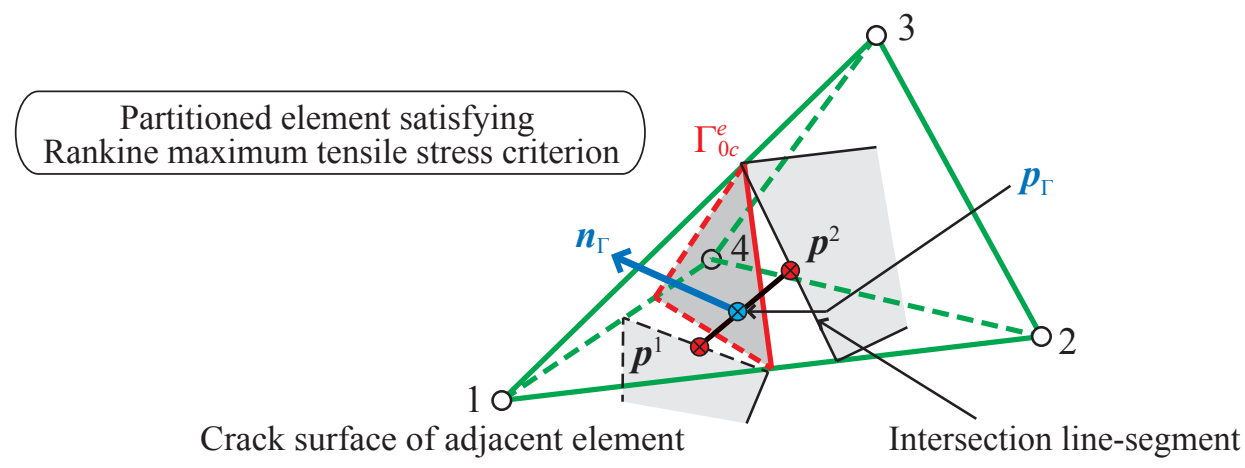

Figure 3: The orientation and the position of "new" crack/discontinuity propagating through elements after modified Rankine maximum principal stress criterion.

Crack passing through nodes:. Though extremely rare, there are cases where the crack plane intersects an element at its node(s). This situation results in non-unique nodal displacement, which leads to the failure of the property of displacement interpolation (see, Equations (9) and (10)). To overcome this issue, a small neighborhood $\Psi_{k}$ is defined around the (intersection) node $k$, as illustrated in Figure 4. Intersection points between crack plane and element edges that fall inside this small neighborhood $\Psi_{k}$ are forced to move (away from the node) to the boundary of $\Psi_{k}$, such that the nodal displacement interpolation property of Equations $90-(10)$ is again fulfilled.

Clearly, the above scheme alters the overall crack topology. However, this can be minimized by choosing sufficiently small radius (or tolerance) for the small neighborhood $\Psi_{k}$. A value around $10^{-4} \ell^{e}$ is recommended, where $\ell^{e}$ represents the size of the element.

Unstable/reversing crack topology:. In the second case, unstable crack propagation often encounters when the angle between a crack plane and the intersecting face is too small. Crack orientation may change when the crack propagates from one element to another. If the angle between the crack plane and the intersection face is too small, the change in crack orientation across the intersecting face may lead to reversing/unstable crack propagation in the subsequent element when passing through an element face with low-angle intersection, as shown in Figure 5 (top).

To avoid this problem, the orientation of the crack plane within an element is constrained by the following condition:

$$
\theta_{f}=\cos ^{-1}\left(\boldsymbol{n}_{\Gamma} \cdot \boldsymbol{n}_{f}\right) \geq \theta_{\Gamma},
$$

for all intersecting faces of the element, where $\theta_{\Gamma}$ is the smallest allowable intersection angle. If the intersection angle with a particular face is smaller than the minimum allowable angle, $\theta_{f}<\theta_{\Gamma}$, the orientation of the crack is slightly modified such that it becomes parallel to the face, $\boldsymbol{n}_{\Gamma} \equiv \boldsymbol{n}_{f}$ and, consequently, the crack plane will no longer intersecting face $f$, as sketched in Figure 5 (bottom).

\section{Experimental campaign}

A full experimental campaign was conducted to validate the model. The open hole tensile test of a $[90 /+45 /-45 / 90 / 0]_{s}$ composite laminate (regularly used in the industry) was selected for validation. The complexity of the deformation mechanisms during failure of such sample naturally lends itself to the validation of the proposed model.

\subsection{Materials mechanical characterization}

The samples for the mechanical tests were obtained from panels of $300 \times 300 \mathrm{~mm}^{2}$ and $2.7 \mathrm{~mm}$ in thickness. Unidirectional carbon fibers/epoxy resin UD300/M10.1 prepreg sheets were supplied by Hexcel $[$ [58]. Individual ply tensile and double cantilever beam (DCB) tests were performed for model calibration. Finally, laminate panels were manufactured in autoclave from the prepreg sheets with a stacking sequence $[90 /+$ $45 /-45 / 90 / 0]_{s}$. An autoclave cure cycle was applied with a maximum cure temperature of $120^{\circ} \mathrm{C}$ for $60 \mathrm{~min}$ and a pressure of 4 bar. The heating and cooling rates were set to $4^{\circ} \mathrm{C} / \mathrm{min}$. The total curing time was $120 \mathrm{~min}$. Each ply thickness was approximately $270 \mu \mathrm{m}$. In this paper, the different plies will be named "ply $n$ " for $n \in[1,10]$, plies 1 and 10 being the outermost plies, and plies 5 and 6 the central ones with the same $0^{\circ}$ orientation.

Twelve open hole specimens of $200 \times 30 \times 2.7 \mathrm{~mm}^{3}$ (length $\times$ depth $\times$ thickness) were machined from the panels with the external plies at $90^{\circ}$ with respect to the length axis. Very little damage was introduced during machining. Steel tabs were fixed to the specimens borders to avoid damaging the samples with the jaws. The distance between tabs was $120 \mathrm{~mm}$. The hole diameter was $6 \mathrm{~mm}$. Tensile tests were carried out in an electromechanical universal testing machine (Instron 3384) at constant cross-head speed of $2 \mathrm{~mm} / \mathrm{min}$. Load was monitored with a $150 \mathrm{kN}$ load cell. The strain was measured with an extensometer with $50 \mathrm{~mm}$ gage length. In addition, strains were monitored by digital image correlation (DIC). An artificial speckle pattern was created with black and white paints in order to monitor de displacements on the specimen's surface. A commercial DIC system from Correlated Solutions, model VIC-2D 2009 was used for this purpose. Both techniques provided equivalent results.

Three specimens were loaded monotonically until failure to determine the average failure stress and strain. The remaining nine specimens were separated in three groups and loaded up to 50,75 and $90 \%$ of the ultimate tensile stress, respectively. The 

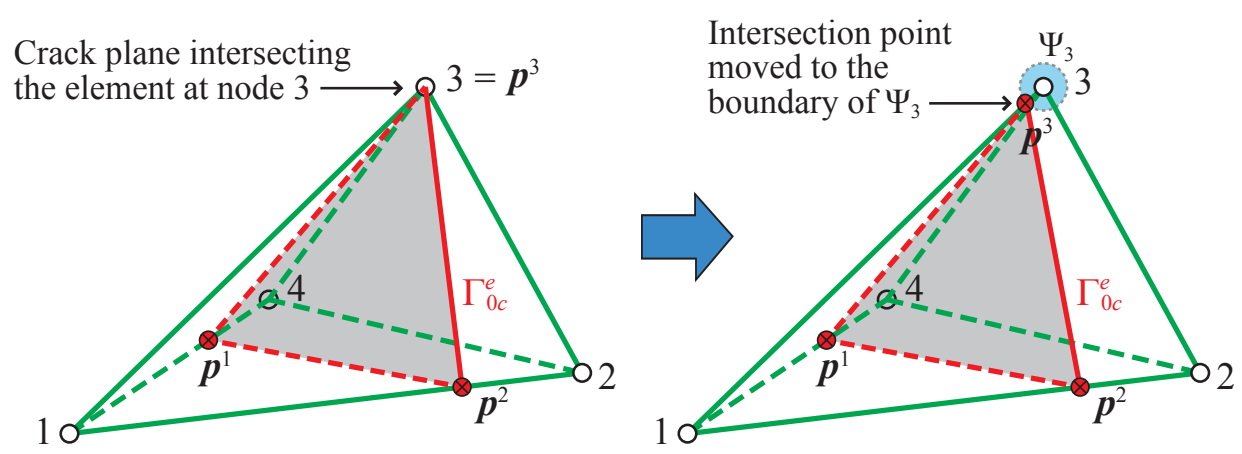

Figure 4: Crack plane intersecting an element at one of its node: this intersection point is moved to the boundary of small neighborhood $\Psi_{k}$.
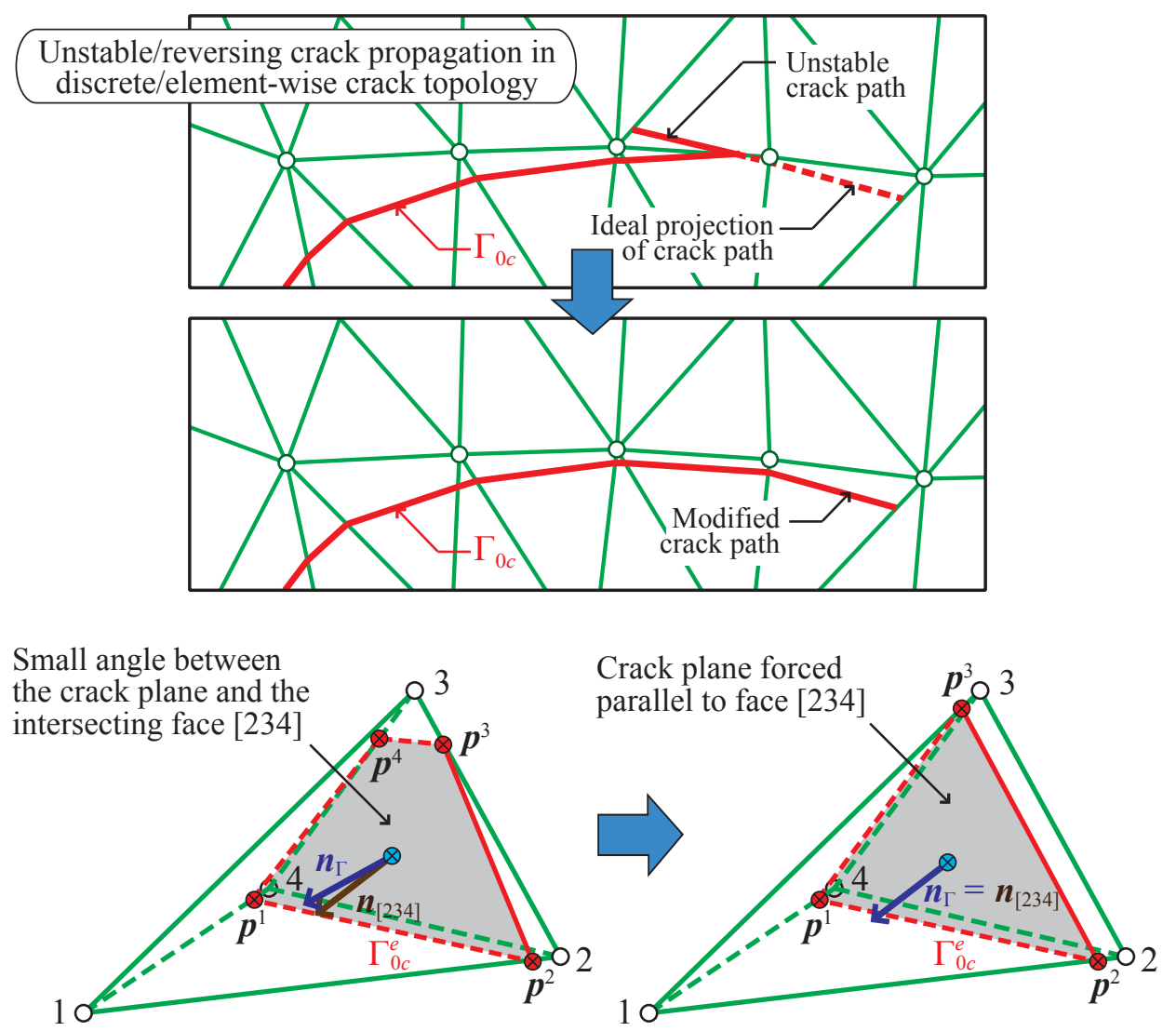

Figure 5: Unstable/reversing crack propagation due to change in crack orientation (top): orientation of the crack plane is modified to avoid unstable crack path (bottom).

tests were stopped at the set conditions and one specimen from each group was immersed in a dye penetrant liquid during 30 minutes while holding the displacement constant. The dye penetrant procedure enhance the contrast between the cracks and the composite material for tomographic measurements. The liquid was composed of $60 \mathrm{~g}$ of $\mathrm{ZnI}$ in $10 \mathrm{ml}$ of water, $10 \mathrm{ml}$ of ethanol and $10 \mathrm{ml}$ of Kodak Photo-Flo 200. The specimen was removed from the machine and inspected by XCT as detailed below. It should be noticed that the stress-strain curve of all the specimens were practically superposed.

\subsection{X-ray Computed Tomography}

The spatial distribution of the failure mechanisms was studied by XCT using a Nanotom 160NF (GE Sensing \& Inspection Technologies Phoenix - X-ray). The tomograms were collected at $100 \mathrm{kV}$ and $120 \mu \mathrm{A}$ using a tungsten target. For each tomogram, 2,000 radiographs were acquired with an exposure time of $500 \mathrm{~ms}$. Tomogram voxel size was set to $15 \mu \mathrm{m}$. The tomograms were then reconstructed using an algorithm based on the filtered back-projection procedure for Feldkamp cone beam geometry. The damage in the reconstructed volumes was qualitatively and quantitatively analyzed using the freeware ImageJ 
software and the commercial software VGStudio Max 2.0. Accurate quantification of crack density and delaminated area was possible because of the use of a dye penetrant liquid containing $\mathrm{ZnI}$ which caused the cracks and delaminations to appear brighter in the tomograms due to the higher X-ray absorption coefficient of $\mathrm{ZnI}$ as compared with the carbon fibers or the polymeric matrix.

Damage at the interface between plies was evaluated from several slices constituting that interface. The information of delaminated area was obtained from superimposed information of these slices by projecting the maximum gray level (brightest) into one plane. The delaminated area was manually segmented at each interface and for each loading condition.

The individuals plies at different orientations showed different cracking patterns. The cracks were properly detected thanks to the dye penetrant technique and were therefore, quantified in each ply. Contrast fading between matrix and cracks occurs when cracks with openings less than the tomographic resolution are filled with the dye-penetrant. However, according to Ref. [46], crack openings of about 5\% of the reconstructed voxel size can be detected using the dye penetrant technique. The selected intralaminar cracks were used to quantify matrix crack density in each ply.

\section{Results}

In this section, the computational model is calibrated against experimental results and validated by direct quantitative comparison between the experimental and simulated interlaminar and intralaminar failure. Finally, the scalability of the code is demonstrated.

The stress-strain curves obtained from the open hole tensile test of the carbon fiber laminates are shown in Figure 6 The strain was determined using both the recording from the extensometer and the DIC data. The response was identical in both cases. The average ultimate tensile stress determined from three samples was $479 \pm 17 \mathrm{MPa}$. The curves are quasi-linear up to the failure, although damage was already observed at $50 \%$ of the ultimate tensile stress (see damage analysis below). The linear behavior of the curves is mainly due to the $0^{\circ}$ plies in which fiber fracture doesn't occur until full sample failure. The tests up to $50 \%, 75 \%$ and $90 \%$ of the ultimate stress are also included in Figure 6, confirming the high reproducibility of the test.

The deformation maps obtained from the DIC measurements at selected deformation conditions in the loading direction are presented in Figure 7. The regions around the hole where the strain ultimately localizes in the outer ply corresponds to the final fracture location, see Figures $7 \mathrm{c}$ and $7 \mathrm{~d}$. Similar strain localization patterns in open hole specimens have been observed previously [59, 60], however in panels with different stacking sequences than the one presented in this work. Note also that additional load introduction and alignment issues cannot be fully discarded. Although the DIC technique provides valuable information on the strain field (and fracture location in the external ply), it does not give information on the strain nor the damage mechanisms in the inner plies. Therefore, X-ray tomography

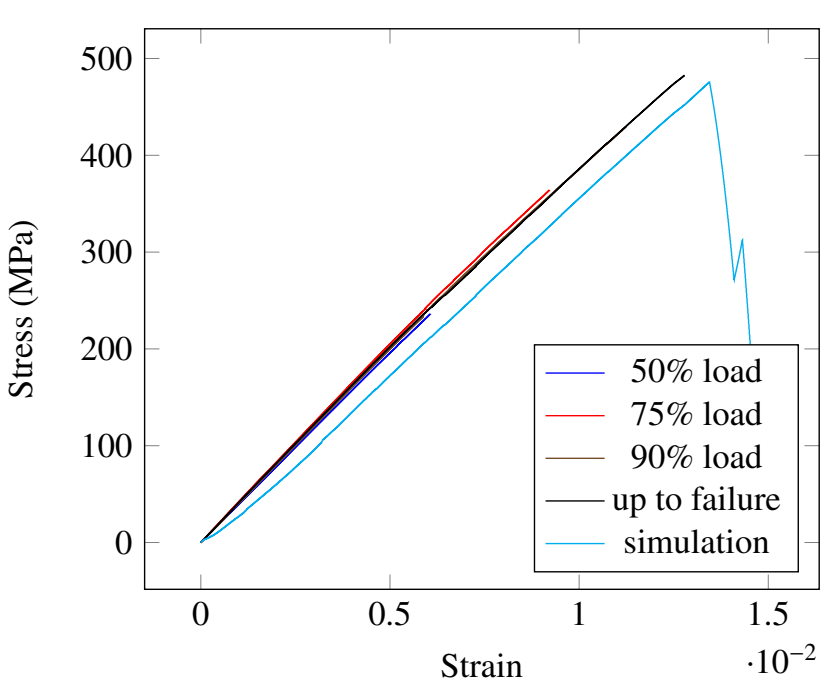

Figure 6: Stress-strain curves for experimental tests and simulation.

was used below to investigate the damage mechanisms inside the specimens.

\subsection{Model calibration}

The transversely isotropic mechanical properties corresponding to each ply of the laminate was obtained from single ply tensile tests. The values of the five elastic constants of the corresponding linear elastic transversely isotropic constitutive model were found to be $E_{l}=139.835 \mathrm{GPa}, E_{t}=8.515 \mathrm{GPa}, v_{l}=0.257$, $v_{t}=0.033$ and $G_{l}=6.3 \mathrm{GPa}$ (respectively, the longitudinal and transversal Young's moduli and Poisson's ratios, and the shear modulus).

The parameters of the two cohesive laws were also calibrated from experiments. For the Rose-Ferrante law (interlaminar intrinsic cohesive model), a DCB test was performed, obtaining a fracture energy $G_{c}=600 \mathrm{~N} / \mathrm{m}$. Using this value, $\sigma_{c}$ was calibrated by simulating the DCB test. A value of $\sigma_{c}=60$ MPa provided the best fit for the force-displacement curve.

The parameters for the intralaminar extrinsic cohesive model were taken from previous work of the authors [31]. In this reference, the calibration was done for the same material by use of a micro-meso-model of intra-laminar fracture which yielded $G_{c}$ $=121 \mathrm{~N} / \mathrm{m}$ and $\sigma_{c}=45 \mathrm{MPa}$ for the overall continuum intralaminar failure, i.e., accounting for both matrix and matrix-fiber failure). Figure 6 shows the numerical stress-strain curve obtained for the model parameters selected, truncated at the strain for which the numerical sample fails, and confirms the validity of these parameters.

In order to compute the number of elements needed for the model, the maximum cohesive element length $\alpha$ was estimated from [16]:

$$
\alpha=\frac{\pi}{8} \frac{E G_{c}}{\sigma_{c}^{2}\left(1-v^{2}\right)}
$$

A good compromise for $\alpha$ for the interlaminar and intralaminar cohesive zones yielded a value of the order of tenths of microns, i.e. 4 million elements were needed for the mesh. Except for 


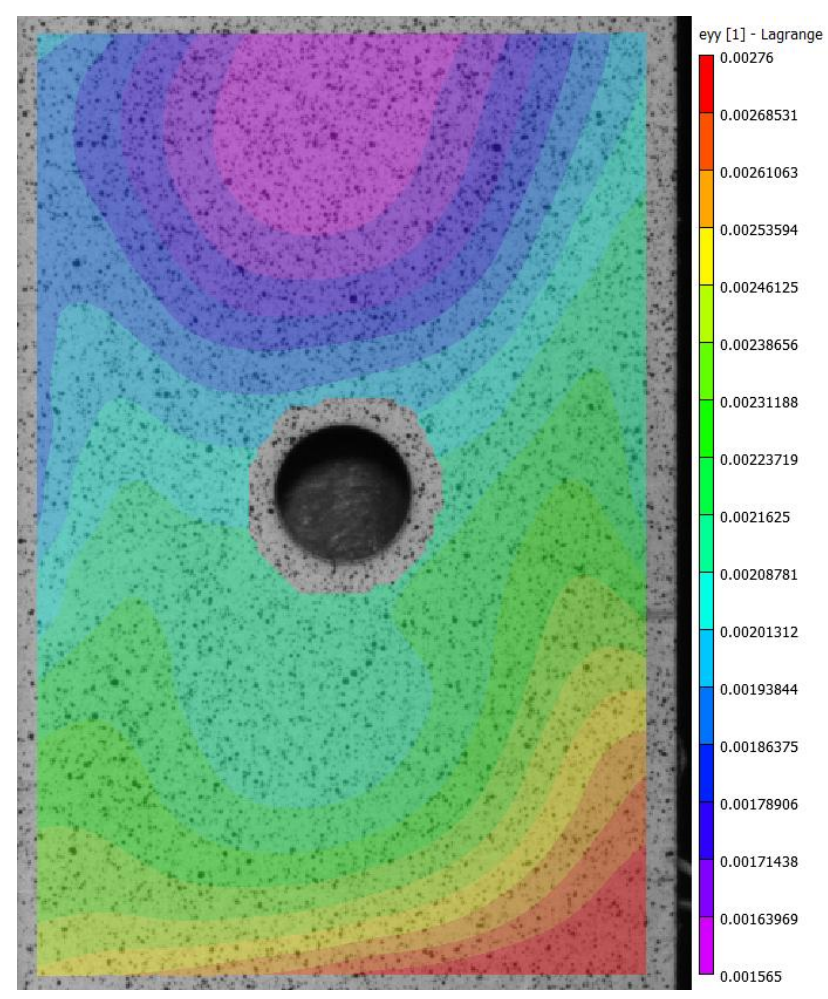

(a) Deformation map at $0.2 \%$ strain

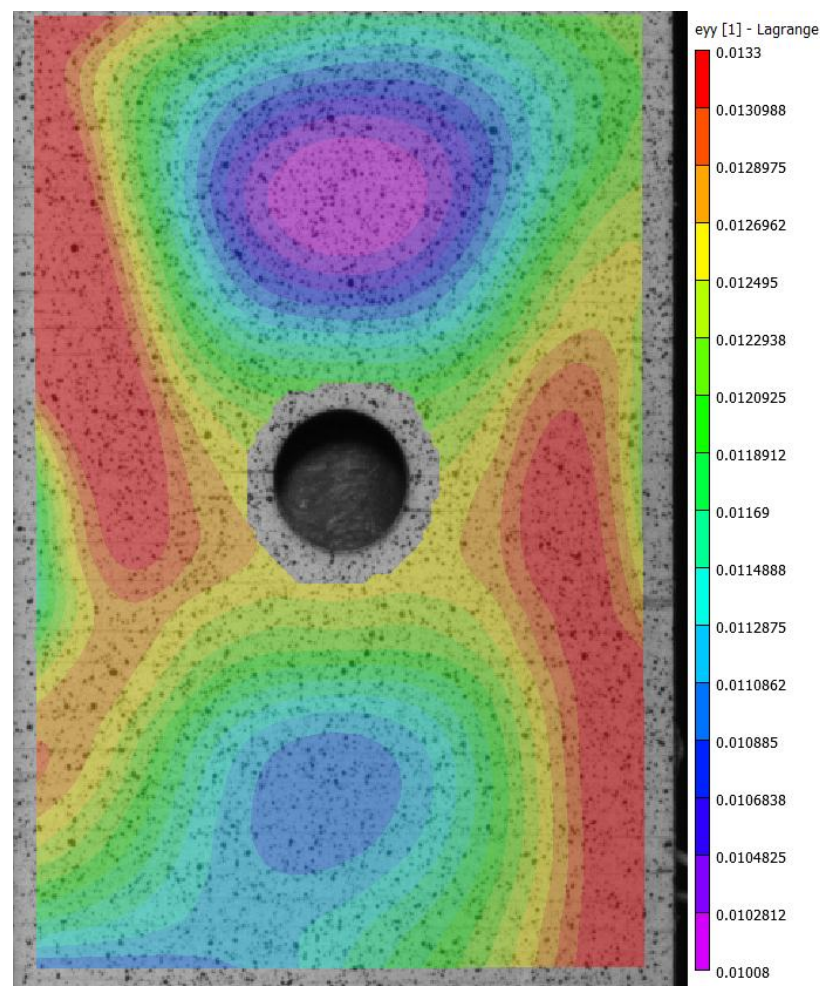

(c) Deformation map at $1.2 \%$ strain

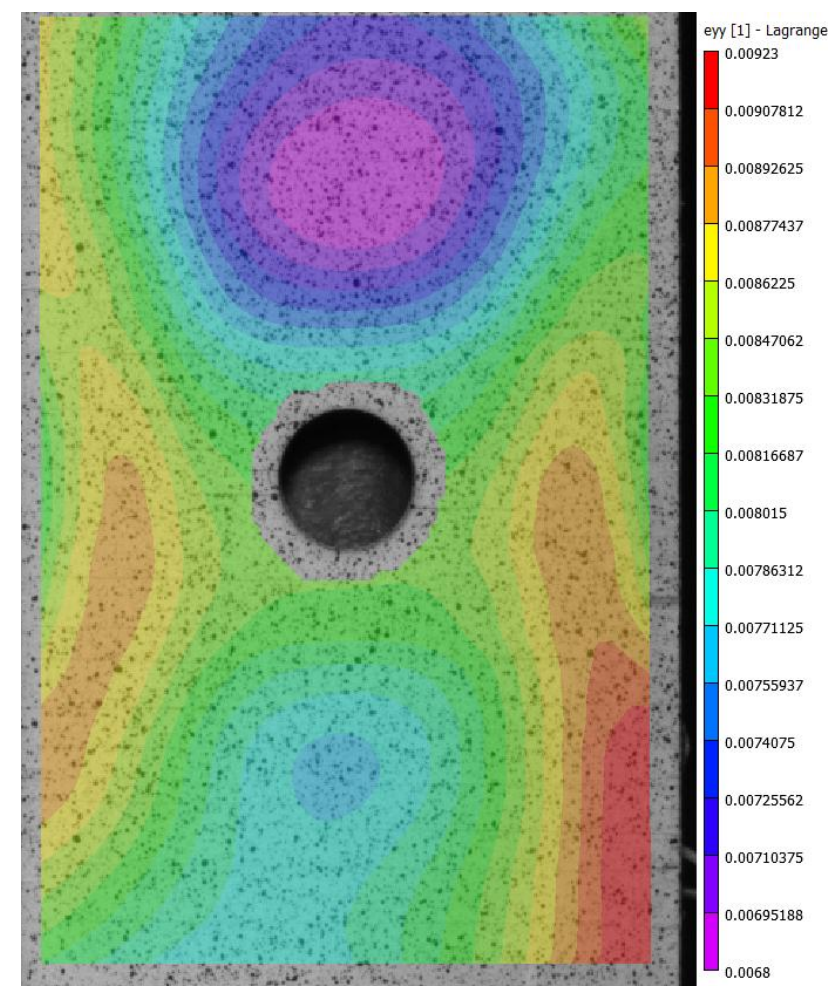

(b) Deformation map at $0.8 \%$ strain

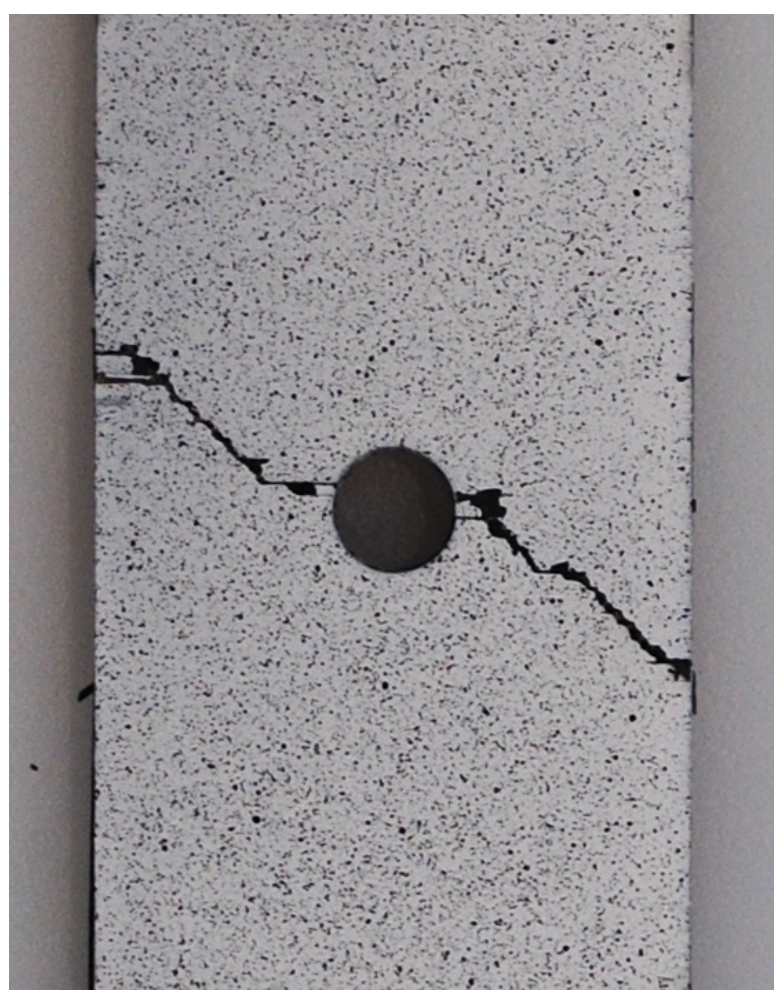

(d) Fractured specimen (at 1.3\% strain)

Figure 7: Deformation maps for several strain conditions and fractured specimen after tensile test (surface with speckle pattern). 


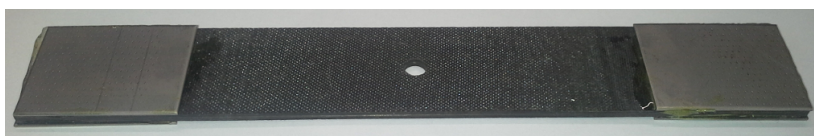

(a) Experimental sample in the initial state

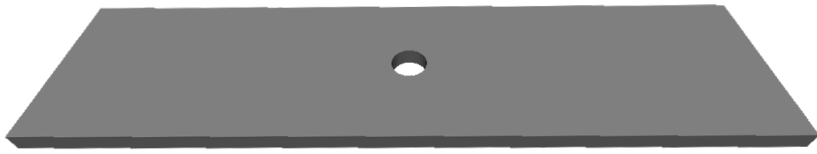

(b) Numerical model used for the simulations

Figure 8: Initial states of experimental sample and numerical model before tensile test.

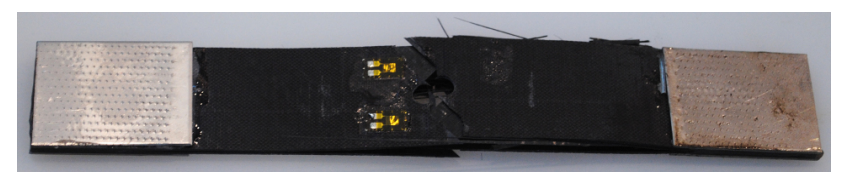

(a) Experimental sample after the tensile test

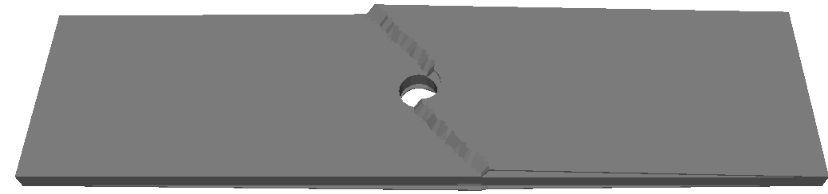

(b) Numerical model after the simulation

Figure 9: Final states of experimental sample and numerical models after tensile test.

the scalability study of Section 5.3 this mesh was used for the subsequent results. Figures $8 \mathrm{a}$ and $8 \mathrm{~b}$ show the initial states of both the experimental sample and the 4 million element numerical model after the tensile test.

Figures $9 \mathrm{a}$ and $9 \mathrm{~b}$ show the states of both the experimental sample and numerical model after the tensile test.

\subsection{Quantitative damage analysis}

Visual inspection of the volumes was performed after reconstruction of the three $25 \%, 50 \%$ and $90 \%$ failure load samples. The three specimens were scanned before loading to identify any damage produced by machining. Very small edge delamination was observed around the hole and at the edges of the specimens, and they were located between the two outermost plies (plies 1 and 2). The initial damage, probably introduced during machining, was symmetrically distributed with respect to the loading axis. Despite the initial damage, the mechanical response of the different specimens was identical and the damage developed during the tests was symmetrically distributed with respect to the central ply (ply 5) as it will be shown later.

\subsubsection{Matrix Cracking}

The inspection of the tomographic data at $50 \%$ failure load ( $0.6 \%$ strain) revealed that cracks appeared in all the plies of the specimens. In the outermost $90^{\circ}$ plies (plies 1 and 9), matrix cracking was observed emanating from the border of the laminate and growing towards the center of the laminate above and below the open hole region following the fiber direction.
On both sides of the hole the cracks were found to already encompass the whole region between the hole and the edge. In the region above and below the hole, matrix cracking then developed with deformation towards the center of the laminate in plies 1 and 9 until they either coalesced at the center of the laminate or stopped growing when no crack grew from the opposite direction at the same position, as it is observed in ply 1 of Figure 10 for $90 \%$ failure load (1.1\% strain).

In the $+45^{\circ}$ plies (plies 2 and 8) short matrix cracks (also called "stitch cracks" to differentiate them from "developed cracks") appeared over the positions of the $90^{\circ}$ cracks. The density of the stitch cracks increased with deformation, see plies 2 and 8 at $90 \%$ failure load in Figure 10. Most of the stitch cracks in the $+45^{\circ}$ plies did not grow in length except for the ones located in a region at $+45^{\circ}$ (following the fibers direction) and containing the hole, as well as some cracks located at the edges of the specimen. The fact that the cracks maintained the same length but increased in number is related to the susceptibility of crack growth to strain energy release rate and the crack spacing in the adjacent and actual ply, as calculated for a $[0 / 60 / 90]_{s}$ and [0/30/90]s laminates in Refs. [61, 62].

Stitch cracks were not observed in the $-45^{\circ}$ plies (plies 3 and 7), see Figure 10 Instead, developed cracks were observed as early as at $50 \%$ failure load, mainly around the hole at $-45^{\circ}$ following the fibers direction and in less proportion at the specimen's edges. With further deformation, the cracks developed mainly in regions located at $\pm 45^{\circ}$ between the hole and at the edge of the specimens, however in these $-45^{\circ}$ plies the cracks were located predominantly at $+45^{\circ}$ from the hole, i.e. in a direction perpendicular to the fiber direction in these plies.

The $90^{\circ}$ plies (plies 4 and 6), located between the $-45^{\circ}$ plies and the central $0^{\circ}$ ply, showed a slower development of the cracks with deformation when compared to the outer $90^{\circ}$ plies. However, the crack density is noticeably higher (smaller crack spacing) than in the outer $90^{\circ}$ plies, see Figure 10 Moreover, a larger concentration of cracks was noticed on both the right and left sides of the hole in comparison with the regions above and below. Even at $90 \%$ failure load, most cracks above and below the hole did not encompass the whole laminate width and were arrested reaching a vertical imaginary line running tangentially to the hole in the loading direction. This effect is related to the damage pattern in the central $0^{\circ}$ ply (ply 5) in which two cracks grew tangentially from right and left of the hole with deformation following the loading direction, see Figure 10

Finally, even if a complete quantitative analysis of the simulations is provided below, it can already be noted that the simulations qualitatively fit very closely the experimental results.

\subsubsection{Delamination}

Figure 11 show the interfaces between the outermost ply and the central ply at $90 \%$ failure load. The images were obtained from superimposed information from the tomographic slices composing the interfaces stacked in the direction perpendicular to the plies. The delaminated area is displayed in Figure 11 by the yellow envelopes delineating the damage.

Delamination between plies was first observed at $50 \%$ failure load around the hole when cracks from subsequent plies 




Figure 10: Matrix cracks for plies 1,2,3,4 and 5 at 90\% failure load. 
(stitch and/or developed) intersected at the ply interface. The delaminated area around the hole increased with deformation and, at $75 \%$ failure load $(0.9 \%$ strain $)$, delamination at the edges of the specimen was noticeable in the outermost interfaces. At $90 \%$ failure load, delamination at the interface 1-2 (between plies 1 and 2), and its symmetric interface 8-9, progressed from the edges towards the interior of the laminate. This delamination was located in the region confined between the $90^{\circ}$ cracks and the stitch cracks in the $+45^{\circ}$ adjacent ply, see Figure 11 .

The second interfaces (interfaces 2-3 and 6-7) showed at $90 \%$ failure load the typical triangular shaped delamination enclosed between the $\pm 45^{\circ}$ cracks at the edges and around the hole, see Figure 11. Most of the delaminated area was located at $+45^{\circ}$ from the hole.

Towards the middle plane of the specimen (interfaces 34 and 4-5) delamination at the edges was less extensive than around the hole. Delamination developed mainly from crack intersection as shown in Figure 11 and was very well correlated to the $0^{\circ}$ ply cracks running parallel to the loading direction and tangentially to the open hole.

Again, the simulation results match very well the experimental results, despite a few differences at interplies 3-4 and 4-5 underneath the hole, probably due to some experimental defects (by the lack of symmetry). It is also noticeable that the boundary effects on the delamination are remarkably well captured.

\subsubsection{Quantitative validation}

Matrix crack density was evaluated for each ply and for the three measured deformation steps. The equivalent nondimensional crack density was selected to quantify the crack density. It was obtained according to Ref. [53]:

$$
\rho_{e q}=\frac{L t}{A}
$$

where $L$ is the total crack length in every ply, $t$ is the ply thickness (since all cracks encompass the whole ply thickness), and $A$ is the in-plane area of the laminate measured by tomography.

The experimental non-dimensional crack density is presented in Figure 12 and shows a very heterogeneous behavior for the different plies. For instance, the outer and inner $90^{\circ}$ plies (1 and 9 , and 4 and 6) showed different behaviors. While the outermost ply saturates after $75 \%$ failure load, the inner $90^{\circ}$ plies can withstand a higher crack density. The highest crack density was achieved in the inner $90^{\circ}$ plies (plies 4 and 6) followed by the density in the $+45^{\circ}$ plies (plies 2 and 8 ) where stitch cracks predominated. The high equivalent crack density observed in the $+45^{\circ}$ plies (plies 2 and 8) suggests that the stitch cracks should have a strong effect on the constraint ply stiffness. The $-45^{\circ}$ plies (plies 3 and 7 ) developed few cracks around the hole and the density in these plies only increased at the end of the test. No stitch cracks were observed in these $-45^{\circ}$ plies. The crack density in the $0^{\circ}$ ply (ply 5) is very low, as expected, and is a consequence of the development of the two cracks located tangentially to the hole, see Figure 12

Figure 13 shows the non-dimensional crack density provided by the simulation. It can be seen that experimental values
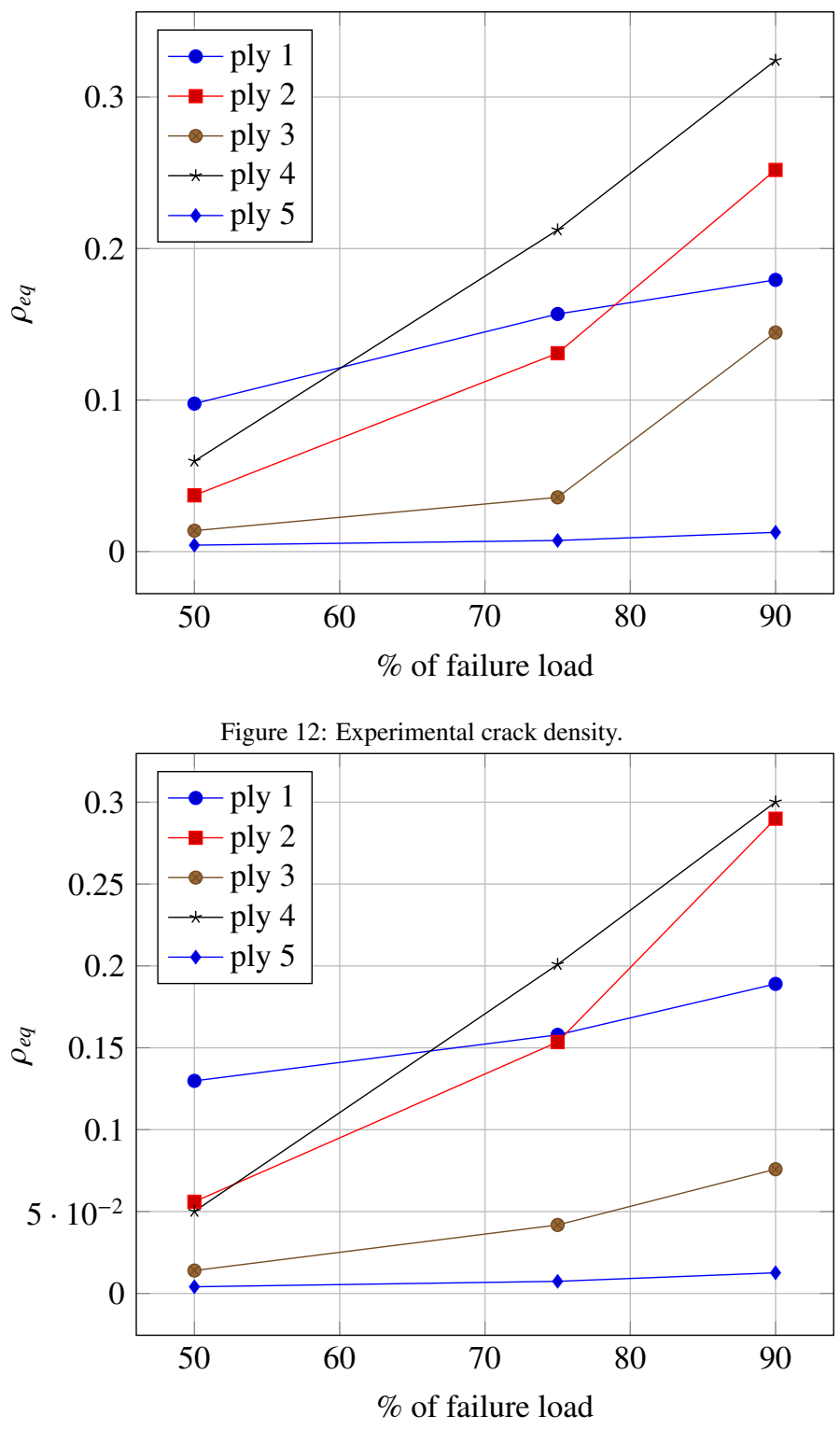

Figure 13: Simulated crack density.

and simulation values are in very good agreement with each other.The curve trend between experiments and simulations is similar and the small differences between experiments and simulations are within the experimental error.

The delamination area obtained from the tomographic volumes is shown in Figure 14. The delaminated area was about $0.1 \%$ at $50 \%$ failure load for all the plies. The development of delamination was symmetric with respect to the middle ply.

At $75 \%$ failure load, the delaminated area reached $1.2 \%$ and was practically the same in all the plies. At $90 \%$ failure load, however, the delamination at the interfaces 3-4 and 6-7 developed faster reaching $8.3 \%$.

Figure 15 shows the area fraction of delamination obtained from simulations, again providing an excellent comparison with the experiments. 


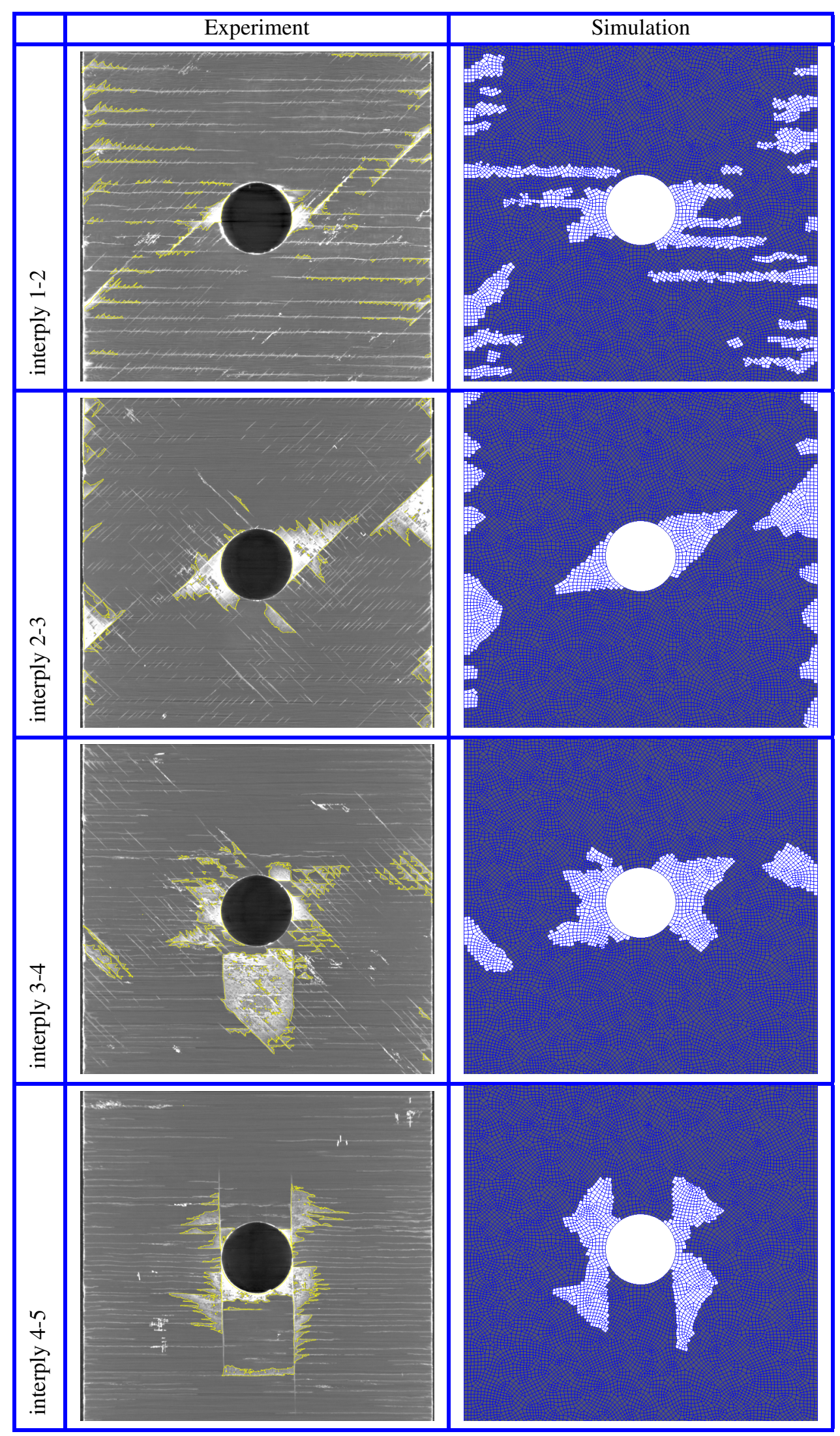

Figure 11: Delamination at 90\% failure load. 

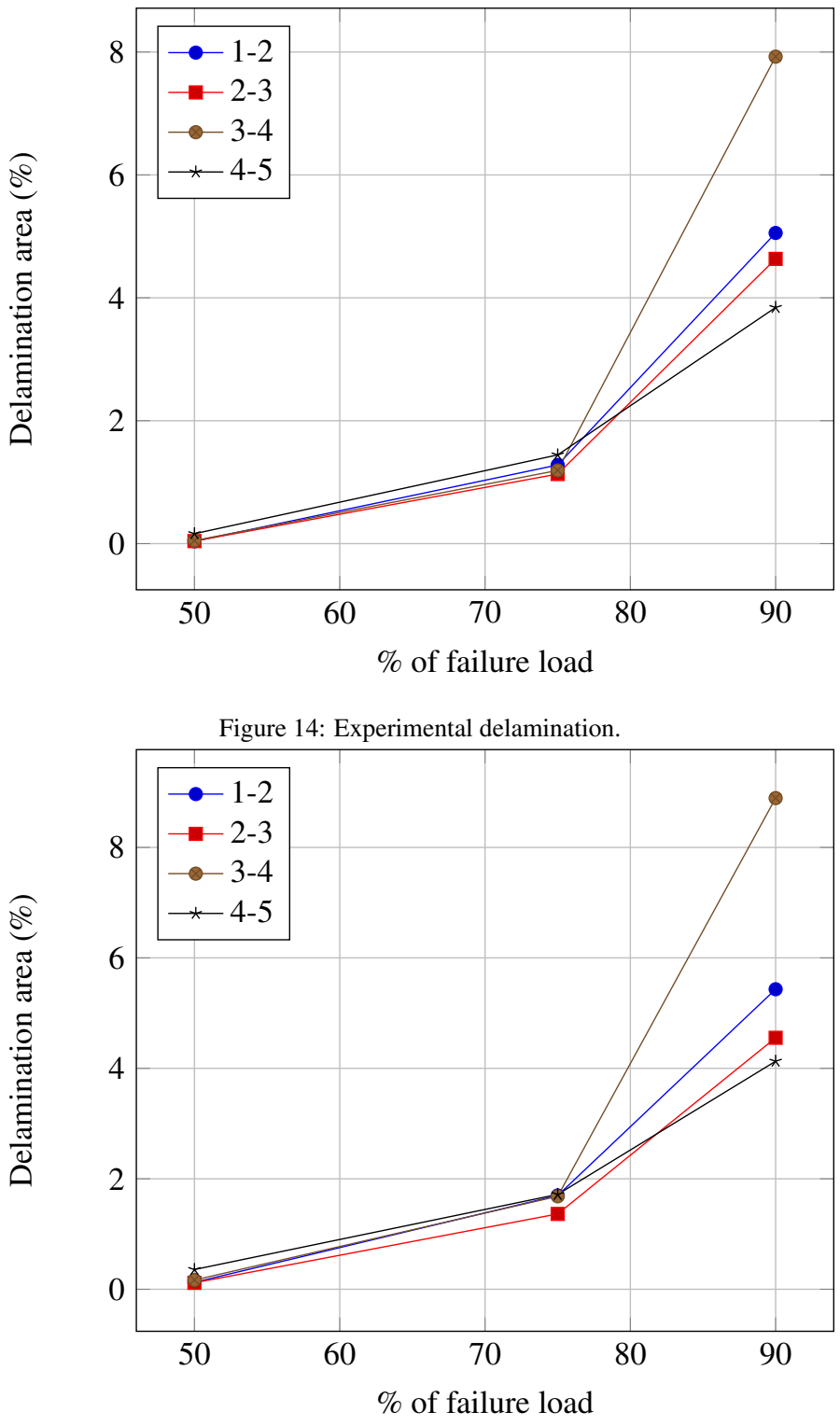

Figure 15: Simulated delamination.

\subsection{Mesh size effect and scalability analysis}

In the following, the parallel implementation performance was evaluated with a convergence study of the XFEM implementation. Note that for this study, interlaminar CZM elements were not introduced in order to evaluate exclusively on the XFEM scalability.

The convergence study was performed by measuring the non-dimensional crack density obtained when using a mesh with different resolutions. To this end, four different resolutions were used: $10 \mathrm{k}$ elements, $100 \mathrm{k}$ elements, $1 \mathrm{M}$ elements and $10 \mathrm{M}$ elements. The results for the previously used $4 \mathrm{M}$ elementmesh (with CZM) are also provided. Figures 16a, 16b, 16c and $16 \mathrm{~d}$ show the density of cracks provided for the three meshes for plies 1,2, 3 and 4, respectively. The crack densities seem bounded and converging. The former property is extremely important as it indicates that the failure of the material is dictated by the energy used to do so, and not by the precision of the ele- ments. Note that, because of the additional dissipation mechanisms used in the corresponding model (interlaminar CZM), the $4 \mathrm{M}$ element-mesh provides a lower crack density and delamination area than its converged intralaminar-only counterparts.

The scalability of the proposed implementation was then evaluated in order to check the performance of the proposed framework for large-scale simulations. Both explicit and implicit simulations using XFEM alone and XFEM combined with cohesive elements were performed. In all cases, the previous 4 million element mesh was used and only the explicit formulation was used for the XFEM/CZM study.

Figure 17 shows the speed-up when simulating the finer mesh with up to 2,048 processors in the explicit case. It can be seen that the proposed implementation provides an excellent scalability since the efficiency is not severely affected when increasing the number of processors. Figure 18 shows that when using the implicit formulation, the performance of the system is reduced. This effect is mainly caused by the scalability of the preconditioners used for solving the system of equations. In this case a Deflated Conjugent-Gradient preconditioner was used and was shown to provide the best scalability results. Although the scalability is reduced when using implicit formulation, the implementation still shows a strong scalability.

Finally, Figure 19 shows that when using cohesive elements the scalability of the system is preserved since it can provide an excellent strong scalability.

Note finally that the maximum number of elements used here is not per se representative of "very large scale" simulations. However, the benefits of large scale parallelization is evaluated here by means of a scalability analysis. Such analysis does not necessarily needs a large mesh, but only needs to exhibit a quasi-linear behavior of the speedup, for an increasing number of processors (and not necessarily a large number number of elements).

\section{Conclusions}

In this paper, a coupled XFEM/CZM parallel implementation of large-scale fracture in composites was proposed. The method was carefully presented and both robustness and scalability were specially targetted by accounting for the non-locality and topological constraints of crack propagation. Both interlaminar and intralaminar failures were shown to be perfectly captured for the open hole tensile test of a quasi-isotropic laminate sequence. The overall framework was shown a) to converge in an energy dependent manner for relatively coarse meshes, and b) to retain the high scalability of Alya, thus paving the road for full component failure simulation in industrial applications.

\section{Acknowledgements}

A.M., D.T., A.J. and L.N. acknowledge funding through SIMUCOMP and ERA-NET MATERA+ project financed by the Fonds National de la Recherche (FNR) of Luxembourg, the Consejería de Educación y Empleo of the Comunidad de Madrid, the Walloon region (agreement no 1017232, CT-EUC 


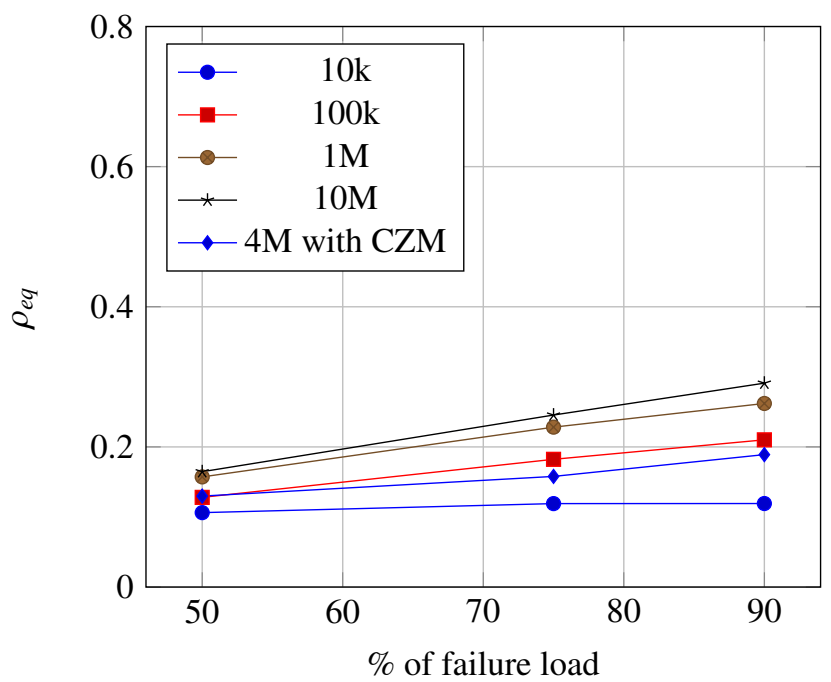

(a) Ply $1\left(90^{\circ}\right)$

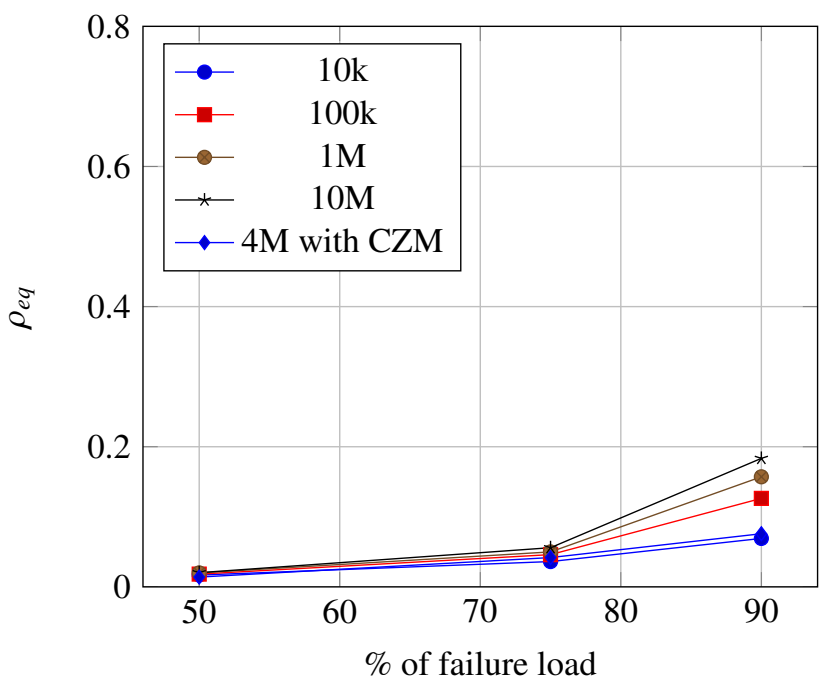

(c) Ply $3\left(-45^{\circ}\right)$

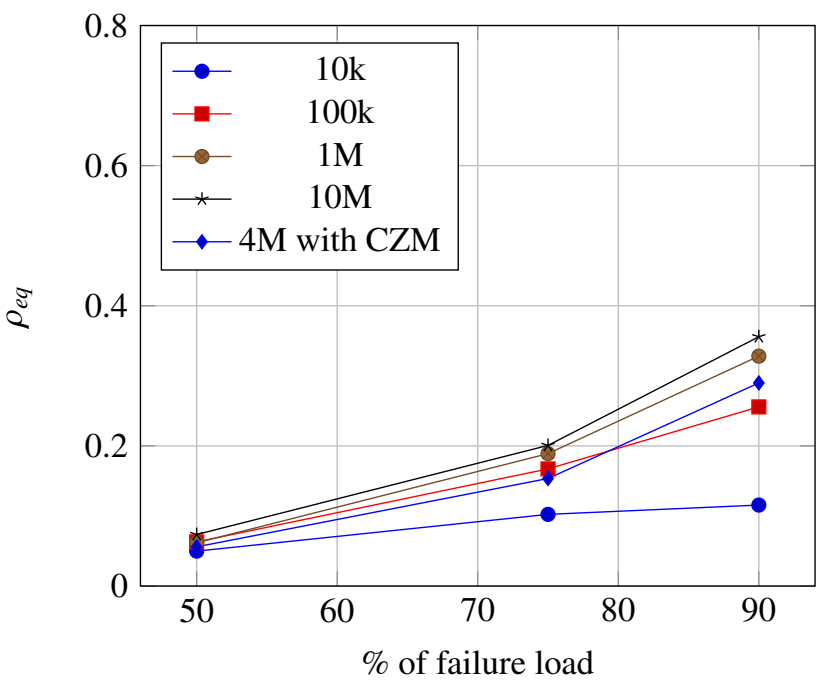

(b) Ply $2\left(+45^{\circ}\right)$

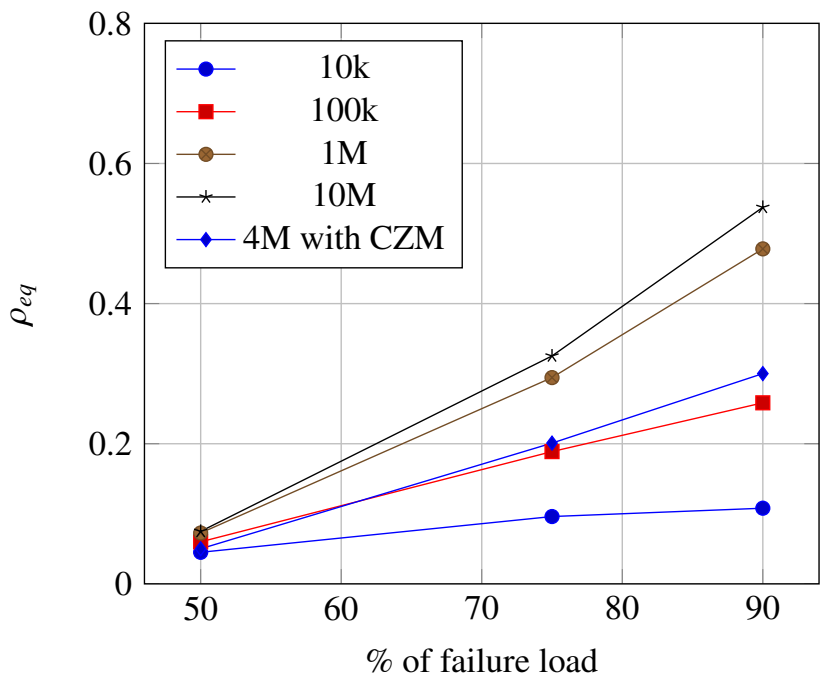

(d) Ply $4\left(90^{\circ}\right)$

Figure 16: Convergence study. 

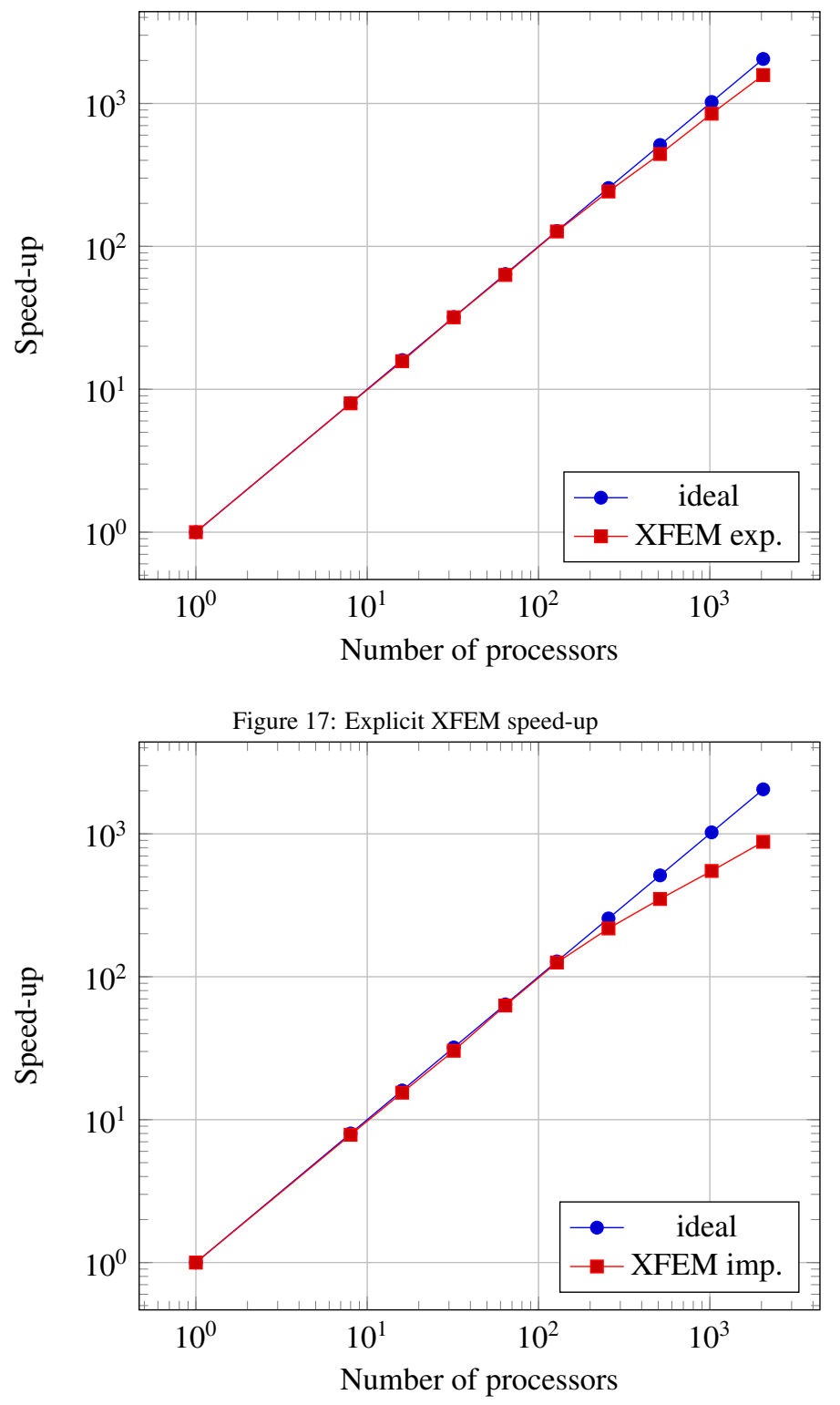

Figure 18: Implicit XFEM speed-up

2010-10-12), and by the European Union's Seventh Framework Programme (FP7/2007-2013).

\section{References}

[1] H. Charles E., J. James H. Starnes, S. Mark J., An assessment of the stateof-the-art in the design and manufacturing of large composite structures for aerospace vehicles, Tech. rep. (2001).

[2] G. Barenblatt, The Mathematical Theory of Equilibrium Cracks in Brittle Fracture Vol. 7 of Advances in Applied Mechanics, Elsevier, 1962, pp. 55-129. doi:10.1016/s0065-2156(08) 70121-2

URL http://dx.doi.org/10.1016/s0065-2156(08) 70121-2

[3] D. Dugdale, Yielding of steel sheets containing slits Journal of the Mechanics and Physics of Solids 8 (2) (1960) $100-104$. doi : http: //dx.doi.org/10.1016/0022-5096(60)90013-2

URLhttp://www.sciencedirect.com/science/article/ pii/0022509660900132

[4] P. H. Geubelle, J. S. Baylor, Impact-induced delamination of composites: a 2d simulation Composites Part B: Engineering 29 (5) (1998) 589

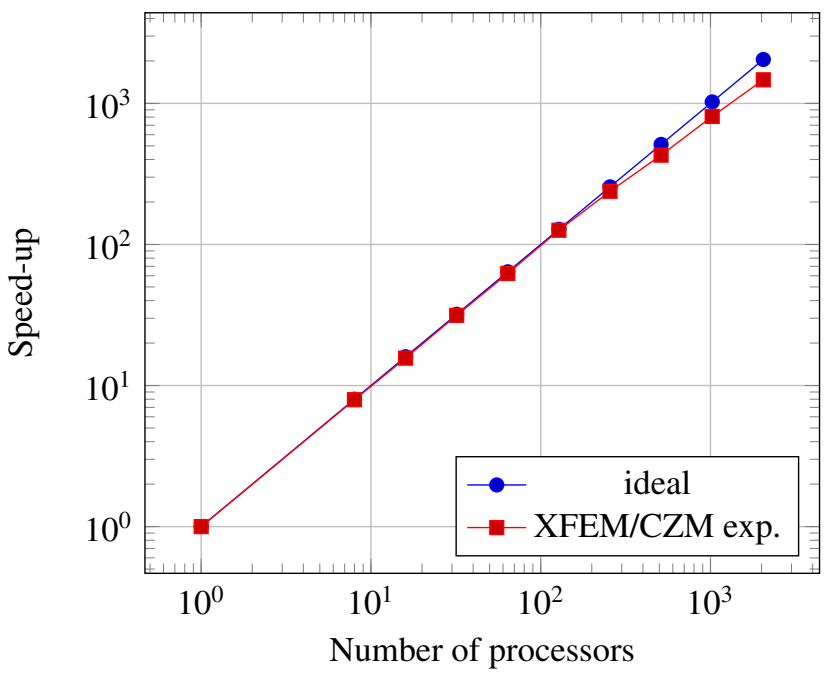

Figure 19: XFEM/CZM explicit speed-up

-602.doi:http://dx.doi.org/10.1016/S1359-8368(98) $00013-4$

URL http://www.sciencedirect.com/science/article/ pii/S1359836898000134

[5] P. P. CAMANHO, C. G. DÁVILA, S. T. PINHO, Fracture analysis of composite co-cured structural joints using decohesion elements Fatigue \& Fracture of Engineering Materials \& Structures 27 (9) (2004) 745-757. doi:10.1111/j.1460-2695.2004.00695.x

URL http://dx.doi.org/10.1111/j.1460-2695.2004. $00695 . \mathrm{X}$

[6] X.-P. Xu, A. Needleman, Numerical simulations of fast crack growth in brittle solids Journal of the Mechanics and Physics of Solids 42 (9) (1994) 1397 - 1434. doi:http://dx.doi.org/10.1016/ 0022-5096(94) 90003-5

URLhttp://www.sciencedirect.com/science/article/ pii/0022509694900035

[7] B. Green, M. Wisnom, S. Hallett, An experimental investigation into the tensile strength scaling of notched composites Composites Part A: Applied Science and Manufacturing 38 (3) (2007) 867 - 878. doi : http: //dx.doi.org/10.1016/j.compositesa.2006.07.008

URLhttp://www.sciencedirect.com/science/article/ pii/S1359835X06002326

[8] S. R. Hallett, W.-G. Jiang, B. Khan, M. R. Wisnom, Modelling the interaction between matrix cracks and delamination damage in scaled quasi-isotropic specimens Composites Science and Technology 68 (1) (2008) 80 - 89. doi:http://dx.doi.org/10.1016/j. compscitech.2007.05.038

URL http://www.sciencedirect.com/science/article/ pii/S0266353807002321

[9] S. H. Song, G. H. Paulino, W. G. Buttlar, A bilinear cohesive zone model tailored for fracture of asphalt concrete considering viscoelastic bulk material Engineering Fracture Mechanics 73 (18) (2006) 2829 2848. doi:http://dx.doi.org/10.1016/j.engfracmech. 2006.04 .030

URL http://www.sciencedirect.com/science/article/ pii/S0013794406001755

[10] N. Blal, L. Daridon, Y. Monerie, S. Pagano, Criteria on the artificial compliance inherent to the intrinsic cohesive zone Comptes Rendus Mécanique 339 (12) (2011) 789 - 795. doi : http://dx.doi.org/ $10.1016 / j . c r m e .2011 .10 .001$ URLhttp: //www.sciencedirect.com/science/article/ pii/S1631072111001677

[11] P. Zavattieri, H. Espinosa, Grain level analysis of crack initiation and propagation in brittle materials Acta Materialia 49 (20) (2001) 4291 4311. doi:http://dx.doi.org/10.1016/S1359-6454(01) $00292-0$

URLhttp://www.sciencedirect.com/science/article/ 
pii/S1359645401002920

[12] G. Camacho, M. Ortiz, Computational modelling of impact damage in brittle materials International Journal of Solids and Structures 33 (20-22) (1996) 2899 - 2938. doi:http://dx.doi.org/10. 1016/0020-7683(95)00255-3

URL/http://www.sciencedirect.com/science/article/ pii/0020768395002553

[13] M. Ortiz, A. Pandolfi, Finite-deformation irreversible cohesive elements for three-dimensional crack-propagation analysis International Journal for Numerical Methods in Engineering 44 (9) (1999) 1267-1282. doi:10.1002/(SICI) 1097-0207(19990330) 44:9<1267:: AID-NME 486>3.0.CO;2-7

URL http://dx.doi.org/10.1002/(SICI) 1097-0207(19990330)44:9<1267::AID-NME486>3.0. $\mathrm{CO} ; 2-7$

[14] A. Pandolfi, P. Guduru, M. Ortiz, A. Rosakis, Three dimensional cohesive-element analysis and experiments of dynamic fracture in $\{$ C300\} steel International Journal of Solids and Structures 37 (27) (2000) 3733 - 3760. doi:http://dx.doi.org/10.1016/ S0020-7683(99)00155-9

URL/http://www.sciencedirect.com/science/article/ pii/S0020768399001559

[15] I. Arias, J. Knap, V. B. Chalivendra, S. Hong, M. Ortiz, A. J. Rosakis, Numerical modelling and experimental validation of dynamic fracture events along weak planes Computer Methods in Applied Mechanics and Engineering 196 (37-40) (2007) 3833 - 3840, ;ce:title ¿Special Issue Honoring the 80th Birthday of Professor Ivo Babuškaj/ce:title $i$. doi: http: //dx.doi.org/10.1016/j.cma.2006.10.052

URL/http://wWw.sciencedirect.com/science/article/ pii/S0045782507001107

[16] J. F. Molinari, G. Gazonas, R. Raghupathy, A. Rusinek, F. Zhou, The cohesive element approach to dynamic fragmentation: the question of energy convergence. International Journal for Numerical Methods in Engineering 69 (3) (2007) 484-503. doi: $10.1002 / \mathrm{nme} .1777$

URL http://dx.doi.org/10.1002/nme.1777

[17] A. Pandolfi, M. Ortiz, An efficient adaptive procedure for threedimensional fragmentation simulations Engineering with Computers 18 (2) (2002) 148-159. doi:10.1007/s003660200013 URL http://dx.doi.org/10.1007/s003660200013

[18] A. Mota, J. Knap, M. Ortiz, Fracture and fragmentation of simplicial fi\begin{tabular}{|l|l|}
\hline nite element meshes using graphs & International Journal for Numerical \\
\hline
\end{tabular} Methods in Engineering 73 (11) (2008) 1547-1570. doi:10.1002/ nme.2135

URL http://dx.doi.org/10.1002/nme.2135

[19] G. H. Paulino, W. Celes, R. Espinha, Z. J. Zhang, A general topologybased framework for adaptive insertion of cohesive elements in finite element meshes Eng. with Comput. 24 (1) (2008) 59-78. do i: $10.1007 /$ s00366-007-0069-7

URL http://dx.doi.org/10.1007/s00366-007-0069-7

[20] V. K. Goyal, N. R. Jaunky, E. R. Johnson, D. R. Ambur, Intralaminar and interlaminar progressive failure analyses of composite panels with circular cutouts Composite Structures 64 (1) (2004) $91-105$. doi: http://dx.doi.org/10.1016/S0263-8223(03)00217-4 URL/http://www.sciencedirect.com/science/article/ pii/S0263822303002174

[21] L. Daudeville, O. Allix, P. Ladevèze, Delamination analysis by damage mechanics: Some applications. Composites Engineering 5 (1) (1995) 17 - 24. doi:http://dx.doi.org/10.1016/0961-9526(95) 93976-3

URL http://www.sciencedirect.com/science/article/ pii/0961952695939763

[22] P. Ladeveze, A damage computational approach for composites: Basic aspects and micromechanical relations Computational Mechanics 17 (12) (1995) 142-150. doi:10.1007/BF00356486

URL http://dx.doi.org/10.1007/BF00356486

[23] J. Mergheim, E. Kuhl, P. Steinmann, A hybrid discontinuous galerkin/interface method for the computational modelling of failure Communications in Numerical Methods in Engineering 20 (7) (2004) 511-519. doi:10.1002/cnm.689

URL http://dx.doi.org/10.1002/cnm.689

[24] R. Radovitzky, A. Seagraves, M. Tupek, L. Noels, A scalable 3d fracture and fragmentation algorithm based on a hybrid, discontinuous galerkin, cohesive element method Computer Methods in Applied Mechanics and Engineering $200(1-4)(2011) 326$ - 344. doi:http://dx.doi. org/10.1016/j.cma.2010.08.014

URL http://www.sciencedirect.com/science/article/ pii/S0045782510002471

[25] M. Prechtel, G. Leugering, P. Steinmann, M. Stingl, Towards optimization of crack resistance of composite materials by adjustment of fiber shapes Engineering Fracture Mechanics 78 (6) (2011) 944 - 960. doi : http: //dx.doi.org/10.1016/j.engfracmech.2011.01.007 URL http://www.sciencedirect.com/science/article/ pii/S0013794411000117

[26] L. Noels, R. Radovitzky, A general discontinuous galerkin method for finite hyperelasticity. formulation and numerical applications International Journal for Numerical Methods in Engineering 68 (1) (2006) 6497. doi:10.1002/nme.1699

URL http://dx.doi.org/10.1002/nme.1699

[27] A. Ten Eyck, A. Lew, Discontinuous galerkin methods for non-linear elasticity. International Journal for Numerical Methods in Engineering 67 (9) (2006) 1204-1243. doi:10.1002/nme.1667

URL http://dx.doi.org/10.1002/nme.1667

[28] L. Noels, R. Radovitzky, An explicit discontinuous galerkin method for non-linear solid dynamics: Formulation, parallel implementation and scalability properties International Journal for Numerical Methods in Engineering 74 (9) (2008) 1393-1420. doi:10.1002/nme.2213 URL http://dx.doi.org/10.1002/nme.2213

[29] A. Lew, A. Eyck, R. Rangarajan, Some applications of discontinuous galerkin methods in solid mechanics in: B. Reddy (Ed.), IUTAM Symposium on Theoretical, Computational and Modelling Aspects of Inelastic Media, Vol. 11 of IUTAM BookSeries, Springer Netherlands, 2008, pp. 227-236. doi:10.1007/978-1-4020-9090-5_21 URL http://dx.doi.org/10.1007/978-1-4020-9090-5_ 21

[30] A. T. Eyck, F. Celiker, A. Lew, Adaptive stabilization of discontinuous galerkin methods for nonlinear elasticity: Motivation, formulation, and numerical examples Computer Methods in Applied Mechanics and Engineering 197 (45-48) (2008) $3605-3622$. doi:http://dx.doi. org/10.1016/j.cma.2008.02.020

URL/http://www.sciencedirect.com/science/article/ pii/S0045782508000789

[31] L. Wu, D. Tjahjanto, G. Becker, A. Makradi, A. Jérusalem, L. Noels, A micro-meso-model of intra-laminar fracture in fiber-reinforced composites based on a discontinuous Galerkin/cohesive zone method, Engineering Fracture Mechanics 104 (2013) 162-183. doi:10.1016/j. engfracmech.2013.03.018

[32] G. Becker, L. Noels, A fracture framework for euler-bernoulli beams based on a full discontinuous galerkin formulation/extrinsic cohesive law combination International Journal for Numerical Methods in Engineering 85 (10) (2011) 1227-1251. doi:10.1002/nme.3008 URL http://dx.doi.org/10.1002/nme.3008

[33] G. Becker, C. Geuzaine, L. Noels, A one field full discontinuous galerkin method for kirchhoff-love shells applied to fracture mechanics Computer Methods in Applied Mechanics and Engineering 200 (45-46) (2011) 3223 - 3241. doi:http://dx.doi.org/10.1016/j.cma.2011. 07.008

URL http://www.sciencedirect.com/science/article/ pii/S0045782511002490

[34] G. Becker, L. Noels, A full-discontinuous galerkin formulation of nonlinear kirchhoff-love shells: elasto-plastic finite deformations, parallel computation, and fracture applications International Journal for Numerical Methods in Engineering 93 (1) (2013) 80-117. doi:10.1002/nme. 4381

URL http://dx.doi.org/10.1002/nme.4381

[35] N. Moës, J. Dolbow, T. Belytschko, A finite element method for crack growth without remeshing International Journal for Numerical Methods in Engineering 46 (1) (1999) 131-150. doi:10.1002/(SICI) 1097-0207 (19990910) 46:1<131: : AID-NME726>3.0.CO;2-J URL http://dx.doi.org/10.1002/(SICI) 1097-0207(19990910) 46:1<131::AID-NME726>3.0. $\mathrm{CO} ; 2-\mathrm{J}$ 
[36] N. Moës, T. Belytschko, Extended finite element method for cohesive crack growth Engineering Fracture Mechanics 69 (7) (2002) 813 833. doi:http://dx.doi.org/10.1016/s0013-7944(01) $00128-\mathrm{X}$

URL/http://www.sciencedirect.com/science/article/ pii/S001379440100128X

[37] J. Dolbow, N. Moës, T. Belytschko, An extended finite element method for modeling crack growth with frictional contact Computer Methods in Applied Mechanics and Engineering 190 (51-52) (2001) 6825 6846. doi:http://dx.doi.org/10.1016/s0045-7825(01) $00260-2$

URL http://www.sciencedirect.com/science/article/ pii/s0045782501002602

[38] J. Melenk, I. Babuśka, The partition of unity ÿnite element method: Basic theory and applications. computer methods, Applied Mechanics and Engineering 39 (1996) 289-314

[39] Alya system URL http://www.bsc.es/computer-applications/ alya-system

[40] E. Casoni, A. Jérusalem, C. Samaniego, B. Eguzkitza, P. Lafortune, D. Tjahjanto, X. Sáez, G. Houzeaux, M. Vázquez, Alya: computational solid mechanics for supercomputers, Archives of Computational Methods in Engineering.

[41] M. Vázquez, G. Houzeaux, R. Grima, J. Cela, Applications of paralle computational fluid mechanics in MareNostrum supercomputer: Lowmach compressible flows, in: PARCFD2007, Antalya (Turkey), 2007.

[42] G. Houzeaux, M. Vázquez, R. Aubry, J. Cela, A massively parallel fractional step solver for incompressible flows, JCP 228 (17) (2009) 63166332

[43] G. Houzeaux, R. de la Cruz, H. Owen, M. Vázquez, Parallel uniform mesh multiplication applied to a navier-stokes solver, Computers \& Fluids In Press.

[44] B. Eguzkitza, G. Houzeaux, R. Aubry, H. Owen, M. Vázquez, A parallel coupling strategy for the chimera and domain decomposition methods in computational mechanics, Computers \& Fluids.

[45] F. Sket, A. Enfedaque, C. Alton, C. Gonzalez, J. Molina-Aldareguia, J. Llorca, Automatic quantification of matrix cracking and fiber rotation by x-ray computed tomography in shear-deformed carbon fiberreinforced laminates Composites Science and Technology 90 (0) (2014) 129 - 138. doi:http://dx.doi.org/10.1016/j. compscitech.2013.10.022

URL http: //www. sciencedirect.com/science/article/ pii/S0266353813004223

[46] P. J. Schilling, B. R. Karedla, A. K. Tatiparthi, M. A. Verges, P. D. Herrington, X-ray computed microtomography of internal damage in fiber reinforced polymer matrix composites Composites Science and Technology 65 (14) (2005) 2071 - 2078. doi:http://dx.doi.org/10. $1016 / j . c o m p s c i t e c h .2005 .05 .014$

URL http: / /www. sciencedirect.com/science/article/ pii/s0266353805001879

[47] K. Reifsnider, A. Talug, Analysis of fatigue damage in composite laminates International Journal of Fatigue 2 (1) (1980) 3-11. doi : http: //dx.doi.org/10.1016/0142-1123(80)90022-5

URL http: //www.sciencedirect.com/science/article/ pii/0142112380900225

[48] J. Masters, K. Reifsnider, An investigation of cumulative damage development in quasi-isotropic graphite/epoxy laminates., In: Reifsnider KL, editor. Damage in composite materials: mechanisms, accumulation, tolerance, and characterization. ASTM STP 775 (1982) $40-62$.

[49] J. Tong, F. Guild, S. Ogin, P. Smith, On matrix crack growth in quasiisotropic laminates - i. experimental investigation Composites Science and Technology 57 (11) (1997) 1527 - 1535. doi : http://dx.doi . org/10.1016/S0266-3538(97)00080-8 URLhttp: //www.sciencedirect.com/science/article/ pii/s0266353897000808

[50] W. Marsden, F. Guild, S. Ogin, P. Smith, Modelling stiffnessdamage behaviour of $( \pm 45 / 90) \mathrm{s}$ and $(90 / \pm 45) \mathrm{s}$ glass fibre reinforced polymer laminates Plastics, Rubber and Composites 28 (1) (1999) 30-39. arXiv: http://www.maneyonline. com/doi/pdf/10.1179/146580199322913304 $10.1179 / 146580199322913304$
URL http://www.maneyonline.com/doi/abs/10.1179/ 146580199322913304

[51] L. Crocker, S. Ogin, P. Smith, P. Hill, Intra-laminar fracture in angleply laminates Composites Part A: Applied Science and Manufacturing 28 (9-10) (1997) 839-846. doi : http://dx.doi.org/10.1016/ S1359-835X(97)00036-5 URLhttp://www.sciencedirect.com/science/article/ pii/S1359835X97000365

[52] J. Varna, R. Joffe, N. Akshantala, R. Talreja, Damage in composite laminates with off-axis plies Composites Science and Technology 59 (14) (1999) 2139 - 2147. doi:http://dx.doi.org/10. 1016/S0266-3538(99)00070-6

URLhttp://www.sciencedirect.com/science/article/ pii/S0266353899000706

[53] J. A. Lavoie, E. Adolfsson, Stitch cracks in constraint plies adjacent to a cracked ply Journal of Composite Materials 35 (2001) 2077 - 2097. doi:10.1177/002199801772661362

URL http://jcm.sagepub.com/content/35/23/2077. abstract

[54] P. Johnson, F.-K. Chang, Characterization of matrix crack-induced laminate failure - part i: Experiments Journal of Composite Materials 35 (22) (2001) 2009 - 2035. doi:http://dx.doi.org/10. $1106 / 7 \mathrm{RN} 1-\mathrm{PFBN}-\mathrm{XQR} 9-3 \mathrm{KDK}$

URL http: / / jcm. sagepub.com/content/35/22/2009

[55] M. S., Extended Finite Element Method for Fracture Analysis of Structures, Blackwell Publishing Ltd., 2008.

[56] F. Van Der Meer, L. Sluys, Continuum models for the analysis of progressive failure in composite laminates, Journal of Composite Materials 43 (20) (2009) 2131-2155. doi:10 .1177/0021998309343054

[57] T. Gasser, G. Hozapfel, Modeling 3d crack propagation in unreinforced concrete using pufem, Computer Methods in Applied Mechanics and Engineering 194 (2005) 2859-2896.

[58] Hexcel corporation URL http: / / ww . hexcel.com

[59] F. Pierron, B. Green, M. Wisnom, S. Hallett, Full-field assessment of the damage process of laminated composite open-hole tensile specimens. Part II: Experimental results, Composites: Part A 38 (2007) 2321-2332. doi : $10.1016 / j . c o m p o s i t e s a .2007 .01 .019$

[60] M. Caminero, M. Lopez-Pedrosa, C. Pinna, C. Soutis, Damage monitoring and analysis of composite laminates with an open hole and adhesively bonded repairs using digital image correlation, Composites: Part B 53 (2013) 76-91. doi:10.1016/j.compositesb.2013.04.050

[61] T. Yokozeki, T. Aoki, T. Ogasawara, T. Ishikawa, Effects of layup angle and ply thickness on matrix crack interaction in contiguous plies of composite laminates Composites Part A: Applied Science and Manufacturing 36 (9) (2005) 1229 - 1235. doi : http: / / dx.doi.org/10.1016/ j.compositesa.2005.02.002 URL http: / /www.sciencedirect.com/science/article/ pii/S1359835X05000692

[62] J. Xu, B. V. Sankar, Prediction of stitch crack evolution and gas permeability in multidirectional composite laminates Composites Part A: Applied Science and Manufacturing 39 (10) (2008) 1625 1631. doi:http://dx.doi.org/10.1016/j.compositesa. 2008.07 .003 URL http://www.sciencedirect.com/science/article/ pii/S1359835X08001887 